Imperial/TP/98-99/23

\title{
On the Emergence of Time in Quantum Gravity'
}

\author{
J. Butterfield \\ All Souls College \\ Oxford OX1 4AL \\ and \\ C.J. Ishamp \\ The Blackett Laboratory \\ Imperial College of Science, Technology \& Medicine \\ South Kensington \\ London SW7 2BZ
}

13 December 1998

\begin{abstract}
We discuss from a philosophical perspective the way in which the normal concept of time might be said to 'emerge' in a quantum theory of gravity. After an introduction, we briefly discuss the notion of emergence, without regard to time (Section 2). We then introduce the search for a quantum theory of gravity (Section 3); and review some general interpretative issues about space, time and matter (Section 4). We then discuss the emergence of time in simple quantum geometrodynamics, and in the Euclidean approach (Section 5). Section 6 concludes.
\end{abstract}

\footnotetext{
${ }^{1}$ To appear in The Arguments of Time, ed. J. Butterfield, Oxford University Press, 1999.

${ }^{2}$ email: jb56@cus.cam.ac.uk; jeremy.butterfield@all-souls.oxford.ac.uk

3 email: c.isham@ic.ac.uk
} 


\section{Introduction}

The discovery of a satisfactory quantum theory of gravity has been widely regarded as the Holy Grail of theoretical physics for some forty years. In this essay, we will discuss a philosophical aspect of the search for such a theory that bears on our understanding of time: namely, the senses in which our standard ideas of time, and more generally spacetime, might be not fundamental to reality, but instead 'emergent' as an approximately valid concept on sufficiently large scales.

In taking up this topic, our aim in part is to advertise to philosophers of time an unexplored area that promises to be fruitful. That is: we maintain that beneath the sometimes rebarbative technicalities of the research programmes in quantum gravity, there are conceptual topics that are sufficiently related to current philosophical debates, that philosophers can both contribute to those topics, and have some light shed on their own debates. More specifically, we believe some general philosophical claims about emergence, which we will state in Section 2, are well illustrated by some of these research programmes. Furthermore, by addressing this topic, and illustrating it with these programmes, our discussion will complement the essays by Barbour and Kuchař (this volume).

We need first to introduce our topic: (this will also yield a prospectus of the later Sections). The first thing to note is an ambiguity in 'emergence' and cognate words. In everyday language, 'emergence' suggests a process in time, as in 'the woman emerged from the crowd', or even an evolutionary process, as in 'amphibians emerged from fishes'. But in this essay, we intend 'emergence' in the non-temporal, philosophical sense of, roughly, reduction, or more often, some relation similar to (usually weaker than) reduction; as in 'the mental properties of a person emerge from their physical properties'; or 'the laws of chemistry emerge from the basic equations of physics'.

We shall discuss the notion of emergence (and reduction) in more detail in Section 2. But we can already state the broad idea of the emergence of time. It is that the concept of time within present physical theories - by which we mean Newtonian physics, special relativity, and general relativity -is an approximation within a quite different conceptual framework that is associated with a quite different theory. Arguably, our present theories' concept of time stems from our psychological awareness of the temporal ordering of events - a property that is reflected mathematically in physics by the use of 
the real numbers (a totally ordered system) to represent 'points' in time. In turn, these real numbers are regarded as the 'temporal coordinate' in a four-dimensional differentiable manifold, whose remaining three coordinates represent space. For Newtonian physics, this distinction between 'temporal' and 'spatial' coordinates is a fundamental ingredient in the corresponding theories. In the case of relativity theory (special or general), the distinction is coded in a metric tensor: essentially, a mathematical structure that specifies both spatial lengths and temporal intervals between points in a unified spacetime.

In this context, the idea of the emergence of time is, roughly speaking, that the concept of time as a continuum ordering of events is only an approximation, valid for sufficiently large scales of time and length (and, correspondingly, sufficiently low energies [), to what is expected to be a quite different concept in the, as yet, unknown full quantum theory of gravity. Note that, for brevity, we shall often talk of the 'emergence of time', not always adding 'and of space' or 'of spacetime'; but in the light of relativity's unification of space and time, it is reasonable to expect that both space and time are emergent concepts.

It is already clear that a full discussion of the emergence of time in quantum gravity would be a very daunting task. It would involve probing an 'intellectual space' with three different 'coordinates': which aspects of time are emergent, in which sense, and in which of a range of quantum gravity programmes. A full discussion would be beyond our - and we dare say, anybody's - competence, if all three coordinates are given a wide range of values, which each get a detailed treatment.

It is not just that among the putative aspects of time are metaphysical matters such as the relation of time to causation, and whether there is temporal becoming: matters which are controversial, in themselves ( $c f$. Tooley, this volume) and in relation to present-day physical theories, let alone some future theory of quantum gravity. Also, even while confining ourselves to those aspects of time that are uncontentious parts of our present theories' treatment (as in the above paragraph), the other 'coordinates' are problem-

\footnotetext{
${ }^{4}$ Those who have succumbed to the seductive allure of the archetype of 'eternal recurrence'-for example, in our own time: Nietzsche and T.S. Eliot-would want to model points in time by the points on a circle, rather than the real line.

${ }^{5}$ In a quantum theory, the scales of time or length are related to the inverse of energy via Planck's constant.
} 
atic. As we shall see in Section 2, the general notion of emergence is vague, and contentious. And once we settle on some such notion, there is a wide and disparate range of quantum gravity programmes to consider. Furthermore, most of these programmes face hard conceptual problems (as well as technical ones), that often involve how time is meant to emerge. We shall mention some of these programmes and problems in Section 3; and in Section 4, we discuss some interpretative issues in more detail.

Thereafter, we shall confine ourselves to a fragment of the overall task. Namely, we will consider just two forms of the canonical quantum gravity programme: (i) quantum geometrodynamics, viewed as the general analysis of the Wheeler-DeWitt equation (Section 5.2 -5.4); and (ii) the Euclidean programme, which uses functional integrals of Euclidean 4-metrics to construct specific solutions of the Wheeler-DeWitt equation (Section 5.5).

These programmes are very closely related. Indeed, one could equally well say, individuating 'programmes' rather differently, that the latter is part of the former. Unsurprisingly, therefore, the latter faces much the same conceptual problems as the former. It has, however, distinctive features which merit the separate discussion in Section 5.5.

We should emphasise at the outset that these two programmes are not now among the main current approaches to quantum gravity. Yet there are three good reasons for choosing them here: reasons relating to the present state of the philosophical literature on quantum gravity, and to this volume.

First: the emergence of time is related to the so-called 'problem of time', which has already been discussed by philosophers (for example, [1]); and though this problem crops up in one form or another for all quantum gravity programmes, it has been most studied, and is best understood, for quantum geometrodynamics. Second, and related: Kuchař (this volume) discusses precisely this problem, for quantum geometrodynamics; so our choosing the same programme gives us the opportunity to write a complementary essay.

Our third reason relates to the Euclidean programme, specifically to a model of quantum cosmogenesis made within it by Hartle \& Hawking [2], viz. the 'no-boundary proposal'. A considerable philosophical, as well as popular scientific, literature about the emergence of time has grown up around this proposal. But we believe that much of that literature is marred by not taking sufficient cognizance of the problem of time, which besets the Euclidean programme as much as quantum geometrodynamics. In a nutshell: by ignoring the problem of time, much of the literature makes it look as if 
the no-boundary proposal treats the emergence of time as a process in time, i.e., according to the temporal sense of 'emergence' noted above. And then, unsurprisingly, the no-boundary proposal seems to face conundrums or even contradictions. But these are an artefact of ignoring the problem of time. In other words: we agree that the no-boundary proposal is very problematic: but this is because it must confront the problem of time (to whose solution it makes no contribution), not because of such conundrums - which we believe rather facile.

\section{Emergence in General}

\subsection{Prospectus}

In this Section we discuss emergence in general, without regard to the idea of time emerging. Although space is short, a brief discussion is called for, for two reasons. First, philosophy has no definite and established usage, for 'emergence' and cognate words. 'Emergence' tends to be used as an alternative - usually, a weaker alternative - to 'reduction': a concept, or a theory or some similar item (such as a law or a model) is said to be, not reducible to another such item, but merely emergent from it. But this leaves open what exactly emergence is (not least because 'reduction' also has no definite and established meaning!), and even what are the items between which it holds. So we need to clarify the terms.

Second, and more important, we also want to make some claims about emergence and its relation to reduction: claims which will be illustrated by the quantum gravity programmes discussed in Sections 4 and 5-but which we think are worth stating in general. These claims will follow from some criticisms (presented in Sections 2.2, 2.3) of a familiar, comparatively precise notion of reduction, and an apparently weaker alternative, supervenience.

To keep the discussion short, we will fix on one traditional choice of the items between which relations of reduction, supervenience and emergence hold. Namely: theories, as understood in the syntactic conception (and in formal logic), i.e., understood as sets of sentences closed under deduction. But we believe our arguments and claims hold good, under other choices; in particular, on the main rival conception of theories, viz. the semantic conception according to which theories are classes of models. 
We will begin (Section 2.2) by introducing a precise notion of reduction for theories. It is, in logicians' jargon, 'definitional extension'. Apart from the advantage of precision, this notion has been immensely influential in twentieth century philosophy: in the first place, in logic and foundations of mathematics, and then in philosophy of science. It has been the formal core of many proposed analyses of the relation of reduction between scientific theories. More precisely: on its own, definitional extension is too weakit applies in cases where intuitively there is irreducibility. So analyses of reduction typically add informal clauses, to try and capture the idea that the reduced theory is 'just a part' of the reducing theory, as regards its concepts, explanatory resources etc. We will maintain that such considerations are so heterogeneous that there seems little prospect of a general formal definition of reduction.

This notion of definitional extension will also lead us to an apparently weaker, but also quite precise, notion: supervenience (Section 2.3). Because supervenience is apparently weaker, it has been a favoured candidate, especially in recent metaphysics, for capturing the idea of emergence, understood as a weaker alternative to reduction. But we will point out that whether supervenience is really weaker than definitional extension turns out to be a subtle matter of logic. We will also claim that because supervenience, like definitional extension, is a formal notion, it is sometimes too weak. That is to say: it applies in cases where intuitively a theory is not emergent from another.

In Section 2.4, we spell out the upshot of this discussion for the notion of emergence, as some weaker alternative to reduction. Just as for reduction, the factors that prompt us to talk of emergence are too informal, and vary too much from one case to another, for there to be much prospect of a satisfactory general, formal definition of emergence. And being emergent is even compatible with reduction in our initial, precise (and often too weak!) sense of definitional extension. We will conclude that, rather than seeking such a general definition, we need to bear in mind the variety of ways that theories can be related: in particular, with one theory being in some sense a limit of the other, or an approximation to it.

\subsection{Reduction}


2.2.1 Introducing Definitional Extension The intuitive idea of one theory $T_{1}$ being reduced to another $T_{2}$ is the idea of $T_{1}$ being shown to be a part of $T_{2}$. The notion of definitional extension makes this idea precise in two main ways. First, it focusses on the syntactic conception of theories. This immediately gives a notion of $T_{1}$ being a part of $T_{2}$, viz. when the theorems of $T_{1}$ are a subset of those of $T_{2}$. (This is called $T_{1}$ being a sub-theory of $T_{2}$.) However, one needs to avoid confusion that can arise from the same predicate (or other non-logical symbol) occurring in both theories, but with different intended interpretations. This is usually addressed by taking the theories to have disjoint non-logical vocabularies. Then one defines $T_{1}$ to be a definitional extension of $T_{2}$, if and only if one can add to $T_{2}$ a definition of each of the non-logical symbols of $T_{1}$, in such a way that $T_{1}$ becomes a sub-theory of $T_{2}$. That is: In $T_{2}$, once augmented with the definitions, we can prove every theorem of $T_{1}$. (The definitions must of course be judiciously chosen, with a view to securing the theorems of $T_{1}$.)

That is the principal idea of definitional extension. But there is a second aspect, which concerns the question: which operations for compounding predicates, and perhaps other symbols, does one allow oneself in building the definitions of the terms of $T_{1}$ ? It has been usual in philosophy to consider a very meagre stock of operations, viz. just the logical operations: the Boolean operations of conjunction and negation, and the application of the quantifiers 'all' and 'some'.

Of course, this restriction does not just reflect the influence of logic on analytic philosophy. It also has a deeper rationale, relating to the striking success of studies in logic and mathematics, from the mid-nineteenth century onwards, in showing various pure mathematical theories to be definitional extensions in the above sense, i.e., using just these logical operations, of others. Indeed, by concatenating such deductions with judiciously chosen definitions, one shows in effect that all of classical pure mathematics can in this sense be deduced from the theory of sets. This remarkable result, showing how large an expressive power can be obtained by applying this small stock of logical operations to a small initial family of predicates, is the source of the traditional philosophical restriction to considering just these logical operations.

It is also of course a result whose impact on analytic philosophy, including analytic philosophy of science, cannot be over-estimated! For recall that the founding fathers of analytic philosophy were very concerned with apply- 
ing the axiomatic method, and more generally the tools of modern logic, to the natural sciences, especially physics. The programme of stating physical theories precisely, as theories in a formal language, and of investigating their logical relations, preoccupied such authors as Reichenbach and Schlick. More specifically, the idea of one such theory being a sub-theory-i.e., a definitional extension - of another became the core idea in analytic philosophers' proposals for the relation of reduction between scientific theories.

We will turn to such proposals in a moment. But first, we should note a general point about definitional extension: for it will be needed in Section 2.3. It concerns the stock of operations for compounding predicates etc. that are used in building definitions. Namely: In discussing physical, rather than pure mathematical, theories it is very natural to augment the tiny stock of logical operations, discussed above, with some of the standard operations of mathematics, such as taking derivatives, integrals, orthocomplements, completions etc. This arises from the fact that even quite simple physical theories such as, for example, Newtonian mechanics of point particles interacting just by gravity, are complicated from the viewpoint of logic and foundations of mathematics; simply because such theories use mathematical apparatus (for example, calculus) that is 'high up' in the deductive chain from basic logic and set theory. So when we undertake to express such a theory in a formal language, we naturally envisage adding the theory's distinctive physical vocabulary, and axioms governing it, to a so-called 'underlying logic' that is much stronger than just basic logic and set theory; i.e., to one that contains standard mathematical operations sufficient to yield the mathematical apparatus; for example, calculus, which is needed by the theory.

2.2.2 Definitional Extension in Philosophy of Science The bestknown proposed analyses of reduction, as a relation between scientific theories, are by Hempel and Nagel, in various papers from the 1940s to the 1970s. The standard reference is [3] (Chapter 11):9] Nagel adds to the core idea of definitional extension some informal conditions, mainly motivated by the idea that the reducing theory should explain the reduced theory; and following Hempel, he conceives explanation in deductive-nomological terms. Thus he says, in effect, that $T_{2}$ reduces $T_{1}$ if, and only if:

\footnotetext{
${ }^{6}$ For other references, together with an evaluation of the proposals for reduction in physics see, for example, Spector 四(Chapters 3-4, esp. p. 44).
} 
1. $T_{1}$ is a definitional extension of $T_{2}$; and also

2. In each of the definitions of the terms of $T_{1}$ (which Nagel calls a 'bridge law'), the definiens (in the language of $T_{2}$ ) must play a role in $T_{2}$; so it cannot be, for example, a heterogeneous disjunction.

We need to make three comments on this sort of proposal; (the details of Nagel's clause 2. will not concern us). In effect, the first is a positive comment about definitional extension. The second and third (in the next two Paragraphs) are negative comments: in short, that definitional extension is sometimes too weak for reduction, and that it is sometimes too strong.

We said above that it was very remarkable that in effect all of classical pure mathematics was a definitional extension using just logical operations, of set theory. But it is equally remarkable how many examples of definitional extension there are in physics, at least once we give the reducing physical theory a suitably strong underlying logic (i.e., a rich enough set of operations) so as to 'extend its deductive reach'. Examples arise in almost any case where there is a derivation of one physical theory from another; and there are many such. For example: the elementary theory of equilibrium classical statistical mechanics for a strictly isolated system, which postulates the microcanonical measure on the energy hypersurface, is a definitional extension of the mechanics of the micro-constituents, once we use an underlying logic strong enough to express calculus (of many variables).

This point is worth stressing, since it tends to be obscured by the traditional emphasis in the philosophical literature on the apparent defects of definitional extension as a general analysis, or part of a general analysis, of reduction. Though we now turn to discuss those defects, they should not obscure the remarkable number of deductive interconnections between various pieces of physics.

\footnotetext{
${ }^{7}$ The details, unimportant for the rest of this paper, are as follows. The notion that this theory adds to the mechanics of the micro-constituents is exactly this microcanonical measure. But within the mechanics together with an underlying logic containing calculus, we can define both (i) the measure's domain of definition, and (ii) the measure itself. (i) is just the (Borel subsets of) the surface in phase space of given energy; and so is definable. (ii) is defined by weighting the Lebesque measure $\mu$ on phase space with the gradient $\nabla E$ of the energy $E$; and both $\mu$ and $\nabla E$ are definable. Incidentally: for its empirical adequacy, the theory needs to assume that the micro-constituents do not interact; cf., for example, [5] (p. 36, 42-3, 111.)
} 
2.2.3 Definitional Extension is too Weak The point that definitional extension is sometimes too weak for reduction has been widely recognized. We can express the point in general, as follows. Even if $T_{1}$ is a definitional extension of $T_{2}$, so that (roughly speaking) each of its predicates (or other non-logical terms) is coextensive with a compound predicate built from $T_{2}$ 's vocabulary: there may well be aspects of $T_{1}$, crucial to its functioning as a scientific theory, that are not encompassed by (are not part of) the corresponding aspects of $T_{2}$. For example, one might think that despite the strict (exceptionless) coextensiveness of predicates, $T_{1}$ 's properties are different from those of $T_{2}$ (including $T_{2}$ 's compound properties). Or one might think that $T_{1}$ has aspects to do with explanation, or modelling, or heuristics, that are not encompassed by $T_{2}$ (even taken together with the coextensiveness, given suitable definitions, of predicates).

Clearly, what these aspects of $T_{1}$ that 'outstrip' $T_{2}$ might be, varies from case to case; and being informal, they are typically controversial. But both the general point, and the controversial status of the details, is well illustrated by Nagel's own proposed analysis of reduction, and the subsequent literature. As we mentioned, Nagel's own supplement to definitional extension, clause 2 . above, was motivated by the idea that $T_{2}$ should explain $T_{1}$, where explanation is conceived in deductive-nomological terms. And following Nagel, there were various rival proposals about how to supplement definitional extension. Some authors focussed on ontological issues about property-identity, and thus on the definitions of $T_{1}$ 's terms (the bridge laws): for example, requiring them to be statements of nomological coextensiveness. Other authors focussed on whether reduction must include explanation, and on what is required for an explanation; and so on.

Fortunately, we do not need to enter the controversy about which such supplementary clauses might be right. We need only note two points.

(A) The first relates to our statement in Paragraph 2.2.2 that physics provides many examples of definitional extension. Namely: though this statement may seem contentious, in the light of various doctrines about the 'disunity of physics', we now see that it is not. For such doctrines say, in effect, that whichever supplementary clauses are right, there are few pairs of

${ }^{8}$ But it is, we think, not as widely recognized as the converse 'Feyerabendian' objection that definitional extension is too strong - discussed in the next Paragraph. Smith [6] (pp $30-32$ ) is a laudable exception. 
theories instantiating them-for physics is disunited. And that is compatible with many instantiations of the 'first clause', definitional extension.

To put the same point in other words: physics abounds with examples of definitional extension, provided that the derived theory is conceived narrowly enough that it does not have properties, explanatory resources etc. that 'ride free' of the deriving theory, and so block the derivation. Indeed, our example of classical statistical mechanics was deliberately chosen to illustrate this. Thus we concede that:

1. Whether statistical mechanics reduces to the mechanics of the individual microscopic constituents is much debated (as is whether thermodynamics reduces to statistical mechanics);

2. As regards our example, one can take the view that the microcanonical measure is a distinctively probabilistic concept, and so is merely coextensive with its micromechanical definiens;

3. More generally, for the standard example of temperature: one can take the view that although in elementary statistical mechanics, the temperature of a sample of gas is definable as the mean kinetic energy of the constituent molecules, a more sophisticated treatment shows that temperature indeed has a 'distinctive identity' oustripping this definiens. Its central role is to be a parameter describing a probability distribution, and as such it figures in the laws of statistical mechanics, and its explanations of various phenomena, in many complex ways: prompting the claim that statistical mechanics is indeed not reducible to micromechanics.

Our point is simply that these concessions in no way undermine our example's claim of definitional extension.

(B) Our second point arising from the weakness of definitional extension is simply that the ongoing controversy over how to supplement definitional extension in an analysis of reduction suggests that there may well be no single 'best' concept of reduction - no 'essence' of reduction to be winnowed out by analysis. The discussion of the next paragraph will support this suggestion.

2.2.4 Definitional Extension is too Strong We turn to the idea that definitional extension, and thereby proposals like Nagel's that incorporate it, 
is sometimes too strong for reduction. Again, the idea is widely recognized: Nagel's proposal, and variants that keep his clause 1. (only adjusting his 2.), are often now regarded as too strict. That is: there are intuitive cases of $T_{2}$ reducing $T_{1}$ without such a deduction of $T_{1}$. This sort of objection goes back at least to Feyerabend [7], who gives examples of $T_{2}$ reducing $T_{1}$ while being inconsistent with it. For example, Feyerabend agrees with Nagel that Newtonian gravitation theory reduces Galileo's law of free fall; but he stresses that it is inconsistent with the law, since it says (contrary to Galileo's law) that the acceleration of a body increases as it falls towards the Earth.

Along with many others, we agree with this objection, and the general idea it prompts: that reduction often involves approximation. Indeed, Nagel himself proposed that a case in which $T_{1}$ 's laws are a reasonable approximation to what strictly follows from $T_{2}$ should count as reduction. More generally, various authors have suggested, in the wake of the critique by Feyerabend et al., that reduction often involves $T_{2}$ including some sort of analogue, $T *$ say, of $T_{1}$. They require this analogue to be close enough to $T_{1}$, in such matters as its theoretical properties and the postulates concerning them, and/or its explanatory resources, and/or its observational consequences, that one is happy to say that ' $T_{2}$ reduces $T_{1}$ ' -rather than something indicating a greater 'gap' between them, such as ' $T_{1}$ emerges from $T_{2}$ ', or even ' $T_{2}$ replaces $T_{1}$ ' or even 'the theories are incommensurable'.

Here our deliberate vagueness about the conditions required of $T *$ is intended to signal both that the various authors differ in their formulations, and that controversy continues. Among the standard cases that get studied are the pairs, Newtonian mechanics and special relativity, and thermodynamics and statistical mechanics; (or better, precise fragments of these very broad theories). While some authors cite them as examples of reduction or emergence, on account of such factors as the first theory providing an approximation, or even some sort of mathematical limit, of the second; other authors cite them as examples of replacement or even incommensurability, on account of such factors as the conceptual and explanatory disparities between them.

Again, we fortunately do not need to enter into the detailed formulations, or the controversy. We only need two uncontroversial points arising from this discussion:

1. The fact that definitional extension often seems too strict for reduction 
prompts one to appeal to such concepts as approximation and limiting relations; and more generally, to the reducing theory containing an analogue of the reduced theory. So there are notions of reduction which at least 'come very close' to the idea of emergence.

2. The controversy suggests, as at the end of Paragraph 2.2.3, that there may well be no single 'best' concept of reduction; and so no sharp division between reduction and such concepts as 'emergence', and even 'replacement' and 'incommensurability'.

So much for reduction, approached in terms of definitional extension (using as the stock of compounding operations either just the logical operations or some richer stock including some standard mathematical operations). We turn to supervenience.

\subsection{Supervenience}

Supervenience is a notion that is apparently weaker than definitional extension, but also quite precise. Because it is apparently weaker, it has been a favoured candidate, especially in recent metaphysics, for capturing the idea of emergence, understood as a weaker alternative to reduction. Indeed, since supervenience is in effect an infinitistic analogue of definitional extension, supervenience promises to make the reduction/emergence distinction as sharp as the distinction finite/infinite: or at least it promises to do this, in so far as reduction involves definitional extension. So at first sight, supervenience promises to overcome the pessimism at the end of the last Subsection, about sharply distinguishing reduction and emergence.

But we suspect that this promise is illusory. After presenting the idea of supervenience, we will point out that whether supervenience is really weaker than definitional extension turns out to be a subtle matter of logic. We will also claim that because supervenience, like definitional extension, is a formal notion, it is sometimes too weak. That is: it applies in cases where intuitively a theory is not emergent from another.

So we begin by presenting supervenience. It is normally introduced as follows. One says that one family of properties $F_{1}$ supervenes on another family $F_{2}$, with respect to a given set $O$ of objects (on which both $F_{1}$ and $F_{2}$ are defined), if and only if any two objects in $O$ that match for all properties 
in $F_{2}$ also match for all properties in $F_{1}$. Or equivalently, one defines with the contrapositive: any two objects that differ in a property in $F_{1}$ must also differ in some property or other in $F_{2}$.

However, this definition turns out to be equivalent to an infinitistic analogue of definitional extension. That is, it is equivalent to allowing each definiens used in giving a definitional extension to be infinitely, rather than finitely, long. The idea of allowing an infinite sequence of compounding operations is at first sight mind-stretching; and one worries that technical obstacles, even paradoxes, may be lurking. But if one takes as one's stock of operations, just the logical operations - the case on which the logic and metaphysics literature have concentrated - the idea is tractable, and well understood. And it is indeed equivalent to supervenience as introduced above, given natural ways of making each idea precise. Accordingly, supervenience is sometimes called 'infinitary reduction', where 'infinitary' means 'infinite or finite'; and where 'reduction' just connotes 'definitional extension'-no informal conditions like Nagel's 2. being required.]

In view of this equivalence, metaphysicians have found supervenience attractive for formulating doctrines of emergence (though some might use another word than 'emergence' for the relation, similar to but weaker than reduction, that they are trying to articulate). For supervenience promises to sidestep controversial issues, such as property-identity and explanation, that, as we saw in Section 2.2, one has to address in order to get an analysis of reduction.

However, we shall now argue that this advantage is often illusory. There

\footnotetext{
${ }^{9}$ Here is the idea of the equivalence: suppose that supervenience as normally defined holds. Then for each property $P$ in $F_{1}$, one can construct a definition of it (as applied to $O)$ by taking the disjunction of the complete descriptions in terms of the family $F_{2}$ of all the objects in $O$ that instantiate $P$. This disjunction will indeed be infinite if there are infinitely many ways objects can combine properties in $F_{2}$ while possessing $P$. But in any case, supervenience will ensure that objects matching in their complete $F_{2}$-descriptions match as regards $P$, so that the disjunctive definiens is indeed coextensive with $P$.

${ }^{10} \mathrm{~A}$ standard, relatively uncontroversial example is given by paintings. Most agree that their aesthetic properties (such as 'is well-composed') supervene on a suitably rich set of non-evaluative pictorial properties (such as 'is magenta in extreme top-left corner'). And many who are happy to accept supervenience have been sceptical that a property such as 'is well-composed' could be a finitely long compound of pictorial properties - after all, they point out, we hardly know how to begin writing such a definition, let alone how to improve it and perfect it.
} 
are two problems.

2.3.1 Is Supervenience Weaker? The relation of supervenience to (finite) definitional extension is more problematic than we have said - and than most of the literature takes it to be! It turns out to be a subtle matter of logic whether supervenience really is weaker than definitional extension (and thereby an attractive candidate for the notion of emergence). In fact there are two points to be made here.

First, in the well-understood case where we consider only the logical operations, it turns out that under wide conditions (roughly: restriction to firstorder languages), supervenience is equivalent to definitional extension! This is the content of Beth's theorem, which says that for such languages, explicit definability (i.e. definitional extension) and implicit definability (roughly, our supervenience) are equivalent.

Second, once we consider not only logical operations, but also various standard operations of mathematics - as urged at the end of Paragraph 2.2.1 the relation between supervenience and definitional extension is much less clear. And it is less clear in ways that threaten the idea that supervenience is weaker. For on the one hand, there are in general no well-defined limits to infinite iterations of such operations. And on the other hand, we noted at the end of Paragraph 2.2.2 the power of definitional extension, once we admitted these mathematical operations. ${ }^{12}$

2.3.2 Supervenience can be too Weak for Emergence The second problem is the analogue for supervenience of the objection in Paragraph 2.2.3 to definitional extension, viz. that it is sometimes too weak for reduction.

\footnotetext{
${ }^{11}$ So far as we know, the first paper to spell out this out, as a threat to supervenience, is Hellman \& Thompson's paper on physicalism [8]. They also suggest a reply to the threat. They argue that we generally expect to cast scientific theories in higher-order languages; for example, in order to escape Godel's verdict of the incompletability of firstorder arithmetic. Since Beth's theorem fails in general for such languages, supervenience may yet be weaker than finite definitional extension in the philosophically important cases: for example, for the formulation of physicalism.

${ }^{12}$ Besides, the fact that in a given case one knows no finite definitional extension, and has little idea how to go about constructing one, hardly show that there is none: a finitely long definition might be so long, say a million pages, as to be incomprehensible to the human mind. Thus there might yet be a finite reduction of paintings' aesthetic properties! For more discussion of the 'power of reduction', cf. [9].
} 
That is: Paragraph 2.2.3 objected that $T_{1}$ might be a definitional extension of $T_{2}$, yet not be reducible to $T_{2}$, since it had properties, explanatory resources etc. that outstripped those of $T_{2}$. A corresponding point can be made here: $T_{1}$ might supervene on $T_{2}$, and yet not be emergent from $T_{2}$.

To make this point precisely, we need to allow for the fact that - setting aside, now, the problem of Paragraph 2.3.1 - supervenience is logically weaker than (finite) definitional extension. That is, we need to allow for the fact that supervenience corresponds to there being definitions of $T_{1}$ 's predicates (or other terms) that are each finite or infinite; it does not correspond to there being at least one definition that is infinite. This last one might well call 'mere supervenience'; (or cumbersomely: 'supervenience, but not its special case, definitional extension'). So the point is that $T_{1}$ might supervene on $T_{2}$, and yet not be emergent from $T_{2}$-and this is not just because $T_{1}$ is in fact a definitional extension of $T_{2}$, and not emergent from $T_{2}$. In other words: the point is that $T_{1}$ might 'merely supervene' on $T_{2}$, and yet not be emergent from $T_{2}$.

How exactly this could happen will of course depend on one's views about emergence. For example, if one takes emergence (as against reduction) to require a certain sort of 'gap' between the properties of $T_{1}$ and $T_{2}$, then the point will be that $T_{1}$ might merely supervene on $T_{2}$, without there being such a gap. Similarly, if one instead takes emergence (as against reduction) to require a certain sort of 'gap' between explanatory resources: $T_{1}$ merely supervening on $T_{2}$ might not secure such a gap.

Admittedly, it is hard to give convincing examples of such cases, for two reasons. First, as emphasised in Paragraph 2.3.1, real cases of mere supervenience are hard to come by: for example, in first-order languages, supervenience collapses into definitional extension. Second, it is a vague and contentious matter what sort of 'gap' indicates emergence as against reduction. Indeed, as we saw in Section 2.2: the controversy over the nature of reduction, and especially over the informal clauses (about analogies, limits and approximations between theories) that might supplement or replace the formal notion of definitional extension, suggests that there may be no single 'best' concept of reduction, and no sharp division between reduction and emergence.

Furthermore, this second reason makes us sceptical of the prospects of a general, formal analysis of emergence; (as we were in Paragraph 2.2.3 and 2.2.4, about reduction). For the factors that prompt people to say one the- 
ory is emergent from, rather than reduced to, another-like the 'distinctive identity' of properties, the autonomy of explanations etc. - are exactly the factors that bedevil a general analysis of reduction. In other words, the present problem - that supervenience threatens to be too weak for emergence, because it is too formal to encompass such factors - is likely to face other attempts to capture the idea of emergence, using purely formal methods. So it is a problem that will probably not be sidestepped by changing formal notions. [3]

\subsection{Emergence}

The upshot of this discussion is to give us a heterogeneous picture of emergence. Though 'emergence of $T_{1}$ from $T_{2}$ ' connotes a greater gap between the theories, than does 'reduction', $T_{1}$ can be emergent in various ways. Furthermore, being emergent is compatible with our initial, formal (and often too weak!) notion of definitional extension.

For the sake of Sections 5, we should fill in this picture a bit: that is, give more detail about the variety of relations, of limit and approximation, that can exist between theories. For both limits and approximations, we want to make two points; they are all illustrated by the theories discussed in Section 5 .

1. As to limits, both points are warnings: that technically and conceptually, the situation can be much more subtle than the elementary idea of a mathematical limit tends to suggest. For first, a limit can be singular in the mathematical sense: and when one goes to such a limit, for some characteristic theoretical parameter of a physical theory, the structure of the theory can change radically. Indeed, the standard example of one theory having another as a limit-special relativity having Newtonian spacetime as its $c \rightarrow \infty$ limit - is a case of this: the theoretical structure changes radically, with the spacetime metric becoming degenerate.

2. Second, the relation between two theories often involves more than one limiting process; and these will in general not commute with one

\footnotetext{
${ }^{13}$ For example, we do not think it is sidestepped by formulating supervenience in terms of the semantic conception of theories; nor by replacing supervenience, by some single, but general, limiting relation between the theories.
} 
another. Again, a standard example - the relation between thermodynamics and statistical mechanics - provides cases. (For example, Compagner [10] discusses the relation between the thermodynamic and continuum limits (among others) of statistical mechanics.)

3. Turning to approximation, we should resist the temptation to think that approximation is always directed at showing that a theory is capable (given the validity of the approximation) of predicting or explaining ('saving') some observable phenomenon. For the aim is often instead to illuminate the theoretical content of a complex (perhaps computationally intractable) theory, without regard to observational predictions, even in some very extended sense of observation. (Both the temptation, and its being wrong, are apparent from the discussion of $T *$ in Section 2.2.4: we are tempted to think of the reduced theory $T_{1}$ as more observational than the reducing theory $T_{2}$, so that $T *$ would be an approximation directed at securing observations; but $T_{1}$ might be wholly theoretical, and both it and its analogue $T *$ might be aimed primarily at illuminating $T_{2}$.)

4. We should distinguish various ways in which one theory (or piece of theory) can approximate another. Of course the core idea is that values of physical quantities should be close to each other (more exactly: in certain circumstances, close enough for certain purposes!) But such closeness can obtain in various ways. Here are three broad (not exclusive) ways, which will crop up in Section 5.

- Neglecting some quantities, and arguing that such neglect is justified, for example by analysing the stability under perturbation of one's calculation, is a well-nigh universal practice in physical theorizing. If the neglect is indeed justified, the full theory is approximated by the fragment that neglects some quantities. (An important special case of this is neglecting quantities describing components of some composite system, in favour of collective quantities describing the whole.)

- Instead of selecting some quantities and neglecting others, one might instead select some states. That is: there might be a subset of one theory's state-space, in which each state approximates a state of another theory. 
- Very often, these first two ways are combined: a subset of states approximates states of the other theory, only for some relevant quantities. Typically, such an approximation is defined by an inequality (often a 'much greater than') between complicated quantities, which is to be read as specifying those states that make it true.

\section{The Enterprise of Quantum Gravity}

In order to discuss the emergence of time in quantum gravity, we first need a brief review of what the enterprise of quantum gravity involves. That is the task of this Section; we will end by stressing some ways that our topic differs from emergence elsewhere in philosophy. This will lead in to a more detailed discussion of some interpretative issues about space, time and matter in Section 4.

\subsection{The Planck Scale}

It should be admitted from the outset, that the subject of quantum gravity is exceptionally difficult and problematic, both in regard to mathematical and to conceptual issues. Furthermore, these problems are compounded by the almost complete lack of any unequivocal data to guide attempts to construct such a theory. In this sense, twentieth-century physics is a victim of its own success: the empirical success of general relativity and quantum theory in their present forms means that we lack data bearing on how we might reconcile them (or more generally, replace them).

Furthermore, it is very hard to see how we could get such data. This is because simple dimensional arguments suggest that quantum gravity effects should be important at about a length-scale called the 'Planck length' $L_{P}$ defined as $L_{P}:=\left(\frac{G \hbar}{c^{3}}\right)^{1 / 2}$; where $G$ is Newton's constant of gravitation, which measures the strength of gravity; $\hbar$ is Planck's constant, which is characteristic of quantum theory; and $c$ is the speed of light. The value of the Planck length is around $10^{-33} \mathrm{~cm}$, which is truly miniscule: the diameters of an atom, nucleus, proton and quark are, respectively, about $10^{-8}, 10^{-12}$, $10^{-13}$, and $10^{-16} \mathrm{~cm}$. So the Planck length is as many orders of magnitude from the (upper limit for) the diameter of a quark, as that diameter is from 
our familiar scale of a centimetre!

The same point can also be made in regard to the other so-called 'Planck scales': the Planck time, the Planck energy, and the Planck temperature. These characterize physical regimes at which quantum gravity effects are expected to be important, and-perhaps - where the continuum picture of space and time breaks down. Like the Planck length, their values are such that they are empirically inaccessible except in the very early universe. For example, the Planck time $L_{T}$ is about $10^{-42}$ seconds, and the Planck energy is $10^{19} \mathrm{GeV}$.

\subsection{Some Motivations for Studying Quantum Gravity}

The central task of quantum gravity is to reconcile two of the main pillars of twentieth-century theoretical physics: general relativity and quantum theory. However, these two theories are disparate in significant ways, and they need to be reconciled. More precisely:

1. General relativity is a classical theory of gravity, but - unlike all other classical theories - it does not work within the framework of a fixed space and time, but is rather a theory of space and time. Specifically, general relativity employs a continuous spacetime manifold, endowed with a spacetime metric that determines the spatial lengths and times elapsed along curves in the manifold. From a physical perspective, the curvature associated with this metric is postulated to describe the gravitational field: in particular, its value at any point is dependent on the state of the (classical) matter at that point. This relation is epitomised in the famous Einstein field equations. (Special relativity also postulates a continuous spacetime manifold, but the metric is a special case with zero curvature.)

2. Quantum 'theory' is not a theory per se but is rather a certain mathematical and conceptual framework within which specific theories are constructed. Typically, these deal with various kinds of matter and the forces they exert on each other, including the fundamental forces other than gravity: the electromagnetic, weak and strong forces. Quantum theories are conceptually very different from classical theories: in particular, the state of a system does not assign real-number values to physical quantities but only probabilities for obtaining such values 
if appropriate measurements are made. And, though they share with general relativity the assumption that it is appropriate to coordinatise space and time with real numbers, they do not treat gravity as curvature of the corresponding spaces: they use either Newtonian spacetime (which corresponds to a degenerate spacetime metric), or the flat metric of special relativity.

The fact that general relativity treats matter classically, and gravity as curvature, while our best theories of matter are quantum theories using a flat metric, is enough to show that some sort of reconciliation is needed. But there are also several other, more specific, motivations for seeking a quantum theory of gravity. For example:

(i) Gravity is, at least prima facie, a force like the others, and these have turned out to be quantum in nature.

(ii) There has been considerable success in developing unified, quantumtheoretical descriptions of the non-gravitational forces (the most successful example being the unified theory of the electromagnetic and weak interactions), which naturally prompts the idea that gravity should be unified with them.

(iii) In the 1960's, spacetime singularities - at which the values of physical fields like curvature and matter density become infinite - were proven to be endemic to general relativity. It has long been conjectured that quantum gravity could remove such defects in the classical theory; rather as the intrinsic instability of the classical atom is resolved by the introduction of quantum theory. The main justification for such an optimistic conjecture is the existence of the Planck length $L_{P} \simeq 10^{-33} \mathrm{cms}$ : the hope is that the 'zero-length' spacetime singularities will no longer arise because of quantum gravity effects that set in at this length scale.

(iv) Quantum theory has various internal problems of its own, and it has been suggested that these could be resolved if general relativity is introduced in an appropriate way. A long-standing example of such a problem is the infinities that arise in relativistic quantum field theory from the singular nature of quantum fields at a spacetime point; again, it is the existence of the Planck length that encourages such speculations. 
Another famous difficulty in quantum theory is the notorious 'measurement problem' with its associated idea of a 'reduction of the state vector'. There have been several suggestions that reduction may be a real physical process that is associated in some way with the gravitational field. The key idea is that real reductions may arise for 'large' objects (thus avoiding the paradoxes associated with Schrödinger's infamous cat); and the size of an object is well reflected in the strength of gravitational field it produces.

These motivations are logically compatible, and one might wish to endorse them all. But, in fact, when one tries to develop a detailed theory of quantum gravity, it is extremely hard to implement any of them in a coherent way; and, in practice, the specific approach to quantum gravity that is adopted often depends strongly on which motivations are uppermost in the investigator's mind. Consequently, the quantum gravity community holds many different views of what such a theory should do, and many different approaches have been tried.

At first glance, there may seem to be a reasonable prognosis for the success of such an endeavour. For example, there are various 'quantization' procedures whereby a quantum version of any given classical theory may be constructed; and, therefore, one obvious approach to quantum gravity, is to try to apply such a procedure to the classical theory of general relativity. Another possibility is to construct a quantum theory that is not a quantization of general relativity, but which nevertheless has that theory as a classical limit - superstring theory is a good example of a structure of this type.

But despite much effort, using a variety of approaches and heuristic ideas, it has turned out to be extremely difficult to find a quantum theory of gravity. In particular, the approaches based on techniques that have been successful for the other forces, run aground.

Of course, we cannot give even a brief review of all the attempts, and the remaining live options ${ }^{\text {th }}$, and in this Section, we will confine ourselves to very brief treatments of a few topics. First, we describe two of the main current approaches to quantum gravity. Second, and most relevant to our theme of the emergence of time: we describe how these approaches assume a manifold structure for spacetime, and how this can be questioned. (What

\footnotetext{
${ }^{14}$ Our paper 11] reviews some of these, from a non-technical viewpoint; a more technical review is [12].
} 
this assumption involves will be further discussed in Section 4.) Third, we take note of some ways in which the emergence of time is expected to be very unlike other cases of emergence.

\subsection{The Two Main Approaches}

There are currently two main approaches to quantum gravity, each of which has several variants and more specific forms. The first exemplifies the strategy mentioned above, of starting with a classical theory and quantizing it; the second affords an example of moving in the opposite direction. The two approaches are as follows:

The canonical approach: The basic idea here is first to recast Einstein's general relativity in Hamiltonian form, in which it describes the evolution in time of 3-dimensional geometries, i.e., the geometry of an instantaneous 3-dimensional hypersurface (a spacelike 'slice') of the spacetime manifold (in general, interacting with matter fields). The ensuing Hamiltonian theory is then quantized by adapting to general relativity one or other of the standard quantization procedures that has been successful in quantizing some of the other fundamental forces.

Nowadays, this approach is mostly pursued in a different form, based on ideas of Ashtekar. The idea of 'splitting' spacetime into 3-dimensional slices, and conceiving dynamics as evolution from one slice to another, remains; but the basic dynamical variable is now, not a 3-geometry, but a 3-connection (roughly: a notion of parallelism for lines). This approach has lead to some radically new perspectives on quantum geometry, not least via choosing the basic variables to be loop-integrals of the 3-connection. However, for reasons given in Section 1, this essay will focus on the older form of the approach.

The superstring programme: Here, instead of quantizing general relativity, one starts with a very different theory whose basic items are quantized, 1-dimensional strings (or higher-dimensional extended objects) propagating in a continuum spacetime. There is a certain sense in which normal general relativity emerges as a low-energy limit of this theory; and the hope is that the other fundamental forces of nature are also contained implicitly in the 
structure. Again, for the reasons given in Section 1, we shall not discuss this important programme further in the present essay.

\subsection{Should We Assume a Manifold?}

We turn now to the issue of the extent to which it is appropriate in quantum gravity to assume the same 'continuum' (i.e. manifold) structure for spacetime as that employed in the classical theory of general relativity. The first point to make is that, at least in their current forms, both the above approaches to quantum gravity do indeed assume a manifold. However, in considering this issue in general it is helpful to think of a 'chain' of successively richer structure, that can be added to a 'bare' set of points, to yield the full classical structure of a manifold with a spacetime metric. For example, if $g$ is a Lorentzian metric on a spacetime manifold $\mathcal{M}$, the pair $(\mathcal{M}, g)$ fits naturally into the chain

set of spacetime points $\rightarrow$ topology $\rightarrow$ differential structure $\rightarrow(\mathcal{M}, g)$

where the lowest level (i.e., the left hand end) is a set $\mathcal{M}$ of bare spacetime points (with the cardinality of the continuum), which is then given the structure of a topological space, which in turn is given the structure of a differentiable manifold (only possible - of course - for very special topologies) which is then equipped with a Lorentzian metric to give the final pair $(\mathcal{M}, g)$. Note that a variety of intermediate stages can be inserted: for example, the link 'differential structure $\rightarrow(\mathcal{M}, g)$ ' could be factorized as

$$
\text { differential structure } \rightarrow \text { causal structure } \rightarrow(\mathcal{M}, g) .
$$

Given such a chain, one can often distinguish approaches to quantum gravity (or their specific forms) by 'how far along towards the right' they assume classical spacetime structure. And one can distinguish approaches that match on this question, by differences in their detailed assumptions about this classical structure, or in their treatments of the "right end of the chain': is it a quantization of the given classical structure, or something else again?

Let us briefly illustrate this sort of classification with a comment about each of the two main current approaches; (there will be further illustrations in Sections 4 and 5.). The approaches match on the initial question-they 
both assume a manifold - but they differ on some of the basic features of this manifold, such as its global topological structure and dimension. In a bit more detail:

- In the canonical approach to quantum gravity, a manifold structure is assumed for 3-dimensional physical space. Furthermore, in the classical formalism with which one starts, a manifold structure is also assumed for spacetime, which is taken, topologically, to be the product of a (spatial) 3-manifold and the real line, representing time.

- In the original, perturbative, approach to superstring theory, the oscillating strings are regarded as propagating in a background spacetime, which also is taken to be a manifold, albeit with a dimension that is possibly greater than four (the extra dimensions are thought to be 'rolled up', like the lateral dimension of a cylinder whose diameter is of Planck size, and hence go unnoticed).

This use of a continuum model for spacetime in quantum gravity has been questioned. Some more radical approaches propose that at sufficiently tiny length-scales (sufficiently high energies) the postulate of a manifold breaks down, and we need a quite different structure. In particular, much effort is currently being devoted to finding a non-perturbative form of string theory (the so-called ' $M$-theory') which is generally expected to involve a novel mathematical model for spacetime: for example, there are tantalizing hints that 'non-commutative' geometry may play a central role.

The idea that simple manifold concepts may not apply at small distances was anticipated by Riemann in his famously prophetic Habilitationschrift of 1854:

"Now it seems that the empirical notions on which the metrical determinations of space are founded, the notion of a solid body and of a ray of light, cease to be valid for the infinitely small. We are therefore quite at liberty to suppose that the metric relations of space in the infinitely small do not conform to the hypotheses of geometry; and we ought in fact to suppose it, if we can thereby obtain a simpler explanation of phenomena." [Translated by Clifford, [13] ] 
Here then is a more radical sort of sense in which time, or better spacetime, might emerge. Of course, this radicalism comes with a price. The usual tools of mathematical physics depend so strongly on the real-number continuum, and its generalizations (from elementary calculus 'upwards' to manifolds and beyond), that it is probably even harder to guess what noncontinuum structure is needed by such radical approaches, than to guess what novel structures of dimension, metric etc. are needed by the more conservative approaches that retain manifolds. Indeed, there is a more general point: space and time are such crucial categories for thinking about, and describing, the empirical world, that it is bound to be ferociously difficult to understand their emerging, or even some aspects of them emerging, from 'something else'. (We develop this point slightly in Section 3.5.)

However, we are sympathetic to this radical sort of approach. And there are a number of proposals of this kind. Given the preceding discussion, perhaps the clearest examples are the suggestions to quantize structures that lie to the left of manifold-structure, in the chain above. For example, there has been work on quantizing topological structure [14], and on the quantization of causal sets [15, 16].

On the other hand, it would be precipitate to blame the difficulties into which the conservative approaches (i.e., assuming manifolds) run, on their use of manifolds. For first, precisely because they are conservative, it is easier to develop them and to compare them with existing theories, and thereby to discover their limitations. And second, as we said above, to a significant extent the difficulties arise from the inaccessibility of the Planck scale (at least, in regard to terrestrial experiments), and the associated lack of any specific data to guide theoretical speculations. This leads to our fourth topic.

\subsection{A Philosophical Warning}

This inaccessibility not only implies a lack of data. It is so extreme that it suggests that those aspects of reality that require a theory of quantum gravity for their description hardly deserve such names as 'appearance', 'phenomenon', or even perhaps 'empirical reality'. Here of course our topic - the emergence of time - touches on deep, in part Kantian, themes about empirical knowledge and belief in general: themes which we cannot properly take up here. But we should take note of the Kantian position that it is not merely very difficult to dispense with space and time in thought about the 
empirical world (as we said above): it is downright impossible.

Scientists are understandably sceptical of claims that certain ways of thought are necessary, not least because the history of science gives remarkable examples of the creative, albeit fallible, forging of new concepts. But in reply, it must here suffice to make three comments in regard to the Kantian position. First, one might only require that certain aspects of space and time, such as their being continua, emerge; and a Kantian might allow that these aspects are in principle dispensable (though perhaps: in practice very hard to dispense with!). Second: maybe the extreme inaccessibility just discussed - the miniscule size of the Planck length and time etc.- puts these aspects of reality (i.e., those requiring a theory of quantum gravity) outside the realm of appearance in the Kantian sense.

Third, and more vaguely: we agree that our topic cannot provide an 'ordinary case-study' of emergence. And this is not just because philosophical controversy continues about how to understand one theory as emerging from another; nor just because we are so far from having a satisfactory theory of quantum gravity that one of the two theories needed for a 'case-study of emergence' is lacking. As noted above, our predicament is aggravated by the continuum model of spacetime being so crucial to our normal physical description of the world. So we must be wary of assuming that the emergence of time will be like more familiar cases of emergence. In particular, we must bear in mind that there is far from being a universal scientific agreement on even the overall shape, let alone the details, of a satisfactory theory of quantum gravity. Furthermore, current approaches to quantum gravity face severe conceptual, as well as mathematical, difficulties, and we must be ready for a complex and unfamiliar relation between the conceptual frameworks of our present theories - using the standard notions of space and time - and those of the, as yet, unknown theory of quantum gravity.

\section{Three Interpretative Issues}

In this Section, we discuss in more detail three interpretative issues that were mentioned in Section 3, and to which the emergence of time is closely tied. These topics are: the idea of a 'fixed' theoretical structure; the role of matter in understanding spacetime; and the interpretation of quantum theory. We will discuss them in order in the next three Subsections, each leading in to the 
next; and we will illustrate the first two topics with some classical spacetime theories including general relativity.

\subsection{Fixity}

Saying that a theoretical structure is 'fixed' in a given theory can have several different meanings, but they all have the connotation that the structure is 'given once for all' in the theory. So to avoid confusion, one needs to distinguish these meanings: in fact, we will spell out three. ${ }^{5}$ This is not just a matter of clarifying terms: we shall see in Section 5 that the problem of time - which, as noted in Section 1, is entangled with the emergence of time - arises essentially from the fact that in quantum theory, time is treated as fixed (in all three of the meanings below), while in general relativity it is not - it is an aspect of the physical system.

In short, our three meanings of 'fixed' are: being classical; being nondynamical; and being the same in all models of the theory. As is suggested by the words 'classical' and 'non-dynamical', these meanings will be rather vague. But we will at least distinguish them sufficiently to see that, with one exception, they are mutually logically independent; and that is enough for our present purposes.

The first meaning is common in discussions of quantum gravity. Here, 'fixed' is used to indicate that a structure present in a classical theory is not quantized (in the given approach to quantum gravity). This is especially common in discussions of programmes that proceed by quantizing a classical theory; (recall the discussion of the chain in Section 3.4).

Second, 'fixed' is used to mean 'not subject to dynamical evolution' (often called, for short: non-dynamical). This usage is not peculiar to quantum gravity, but occurs in a variety of discussions of spacetime structure. In this sense, the metrical structure of spacetime is fixed in Newtonian physics and in classical and quantum theories that work within a special relativistic (Minkowski) spacetime. But it is not fixed in general relativity.

This second meaning is clearly logically independent of the first. The case of quantum field theory in a curved spacetime shows that the first meaning of

\footnotetext{
${ }^{15}$ Another source of confusion is that there is no established usage about which word to use. All of the adjectives, 'external', 'prior', 'absolute', 'primitive' and their cognates are sometimes employed. But for various reasons, we prefer 'fixed' and its cognates.
} 
'fixed' - not quantized - does not imply the second, viz. being non-dynamical: for example, the (unquantized) metric tensor might obey a form of Einstein's equations in which the right hand side is an expectation value of the energymomentum tensor of the quantized matter fields. And the converse is also false: a structure can be non-dynamical but quantized. An obvious example is a conserved quantity in standard quantum theory: for example, the total angular momentum in a system (like the hydrogen atom) whose Hamiltonian is spherically symmetric. It is clearly non-dynamical (in the sense that its eigenvectors do not evolve in time; or, equivalently, the corresponding operator in the Heisenberg picture does not change in time.) but it is not classical since it is represented by an operator $\hat{J} \cdot \hat{J}$, and most states do not give it a definite value. ${ }^{\text {Tr }}$

The third meaning of 'fixity' is that a theoretical structure is 'fixed' if it is 'given once and for all' in the very formulation of the theory, i.e. it is the same in all the different descriptions that the theory allows of its subject matter; such structure is often said to be part of the fixed 'background'. So the idea assumes a contrast between (i) the theory, that describes all the patterns of behaviour of its subject-matter that are possible according to the theory; and (ii) an individual pattern. An individual pattern is typically a history of a system of the kind described by the theory, and is called a model; and the theory's main content is a set of dynamical laws (laws of evolution over time) - in physical theories, usually differential equations. So a fixed theoretical structure is common to all such models.

One naturally expects that it would be easier to make this third meaning precise than the first two, about being classical and being non-dynamical. But in fact it is surprisingly hard to make precise sense of a theoretical structure being the same in all models of the theory. It turns out that even when one uses a formal notion of model, and so can precisely define associated notions such as isomorphisms of models, it is difficult to define 'fixed' in such a way as to capture intuitions about what counts as fixed - even in a small

\footnotetext{
${ }^{16}$ Of course, people also use 'classical' to mean a wider notion than 'all quantities having a definite value in all (pure) states'. For example, they say it of any mathematical structure that is used in a classical theory; so that, for example, the assumption of a product manifold structure for spacetime by geometrodynamics (Section 3.3) counts as classical. (And they even say 'fixed' to indicate this wider sense of 'classical'.) But even in this wider sense of 'classical', being non-dynamical does not imply being classical: again, the angular-momentum operator $\hat{J} \cdot \hat{J}$ in elementary quantum theory is an example.
} 
handful of precise and well-understood theories, such as classical theories of electromagnetism and gravity in Newtonian and relativistic spacetimes. In fact, controversy continues about this. [T.

We emphasise that this is not a mere problem of warring intuitionspeople agree about which theoretical structures are intuitively fixed in these theories: for example, that the spacetime metric is fixed in special relativity, but not general relativity. The problem is to frame a definition that captures the intuitions.

This problem is illustrated by the fact that this third meaning is, for the most part, logically independent of the first two. The exception is that the third meaning presumably implies the second. Or in terms of the contrapositive: being dynamical presumably implies being not fixed in the third sense. For it would be an odd theory that has such little variety in the configuration of matter fields, from model to model, as to make such an influence completely uniform, giving fixity in the third sense. But the converse is false: a structure could be unfixed in the third sense, but not dynamical - a sheer happenstance of the world, varying from one model to another but uninfluenced. An obvious example of this is the global topological structure of time in Newtonian theories, written as allowing topologies other than the real line.

And this third meaning is logically independent of the first. Being classical does not imply being fixed in the third sense: $c f$. the metric in general relativity. And the converse implication also fails: for example, in the standard quantum theory of a system moving in 3-dimensional Newtonian space, the algebraic form of the canonical commutation relations $\left[\hat{x}_{i}, \hat{p}_{j}\right]=i \delta_{i j} \hbar$ $i, j=1,2,3$, is fixed in the third sense, but it is not classical since it involves operators.

We do not need to pursue more precise definitions of these three meanings of fixity. But we should make three remarks, in increasing order of importance.

- First, we should note a trivial reason why fixity is vague, which can cause confusion. It arises from the fact that, even in a single theory, a word such as 'time' or 'electromagnetic field' usually stands for a whole cluster of mathematical structures, often hierarchically organized from general (logically weaker) down to specific (stronger). Typically, some

\footnotetext{
${ }^{17}$ The word 'absolute' is commonly used in place of our 'fixed'; a recent discussion, with full references to previous proposals (by authors such as Friedman and Torretti), is [17].
} 
general structures are fixed (in all three of our senses) even if specific ones are not. For example, the fact that the set of all time points has the cardinality of the continuum is fixed (in all three senses) in general relativity, though its metrical, and global topological structure is not (in the second and third senses). Similarly, the differentiable nature of the electromagnetic field (away from point-particle charges) is fixed (in all three senses) in classical electromagnetism. So to say, for example, "time is not fixed in general relativity" is somewhat ambiguous: we are really saying that some aspect of time - contextually determined as being of interest: usually, its metric structure - is not fixed.

- In discussing quantum gravity, one must beware of a temptation to think that the third sense of fixity implies the first: that being the same in all models implies being classical. The temptation arises because it is common in quantum gravity programmes that quantize a classical theory, to take as fixed in the third sense, a structure that is classical. For example, around 1970 there was an (unsuccessful) programme to quantize general relativity by quantizing metric perturbations around the fixed, background spacetime metric of special relativity.

- Finally, we emphasise that the problem of precisely defining the third sense of fixity is closely connected with the diffeomorphism invariance of theories such as general relativity, and thereby with the deep interpretative issue of whether or not, and in what sense, spacetime points are 'real'. Here, 'diffeomorphism invariance' refers to the fact that the physical content of the physical content is wholly unaffected by applying any smooth, invertible transformation (called a 'diffeomorphism') between spacetime points, which thus function as mere 'pegs' on which to 'hang' the fields; this is the force of Einstein's famous 'hole argument'. This feature suggests that-quite apart from difficulties at the scale of the Planck-length - spacetime points should not be taken as real objects, even in interpreting classical general relativity: rather they are an artefact of the way we have formulated the theory. This leads to the next Subsection. 


\subsection{Spacetime and Matter}

In the introduction to quantum gravity in Section 3, we barely mentioned something that everyday thought takes to be much more unproblematically real than space and time: namely, matter! And Section 5 will similarly say little. Here, we will first discuss why matter comes to play second fiddle to space and time; and then we will briefly sound a warning against taking spacetime as in some way more basic than matter.

There are special technical reasons why so little was said about matter in Section 3; and also a deeper, general reason. From a technical perspective, many of the conceptual issues surrounding the emergence of time, or the problem of time, are much the same, whether or not one considers matter. For example, as Kuchař (this volume) says, "matter is not the heart of the problem [of time in geometrodynamics]; it is gravity".

The deeper, more general, reason arises from the way that matter has come to be treated in twentieth-century physics. Namely, some of the most distinctive characteristics of matter, at least as idealized by the mechanical philosophy of the seventeenth century - such as impenetrability and continuityturn out to be only apparent; and others, such as massiveness, turn out to be shared with invisible fields that extend in 'empty space' arbitrarily far from their sources. Further, matter itself has come to be treated as a field, and this involves being represented mathematically by an assignment of a mathematical object - at its simplest: an item like a real number, or a vector, or a matrix - to each point of space or spacetime.

Thus, with the rise of classical field theory, culminating in general relativity, the field gradually became endowed with properties previously thought peculiar to matter. Thus the electromagnetic field was discovered to possess mechanical properties like momentum and angular momentum, and (after the advent of special relativity) mass-energy. The 'action-at-a-distance' of Newtonian gravity readily admits a translation into the language of fields; and, of course, the theory of general relativity was couched in such language from the outset. Furthermore, this assimilation of matter to the concept of a field continued with quantum theory. Even elementary quantum theory has a formulation (wave mechanics) which can be thought of as a field theory. And this picture deepens with the advent of quantum field theory: here physical quantities describing matter are again represented by a field; albeit in a transmogrified form in which an operator (rather than a simple real number) 
is associated with each space, or space-time, point.

This development has of course had as great an influence on the philosophical understanding of space and time, as of matter itself. In short, the effect has been for matter to now play second fiddle to space and time. As these field theories, both classical and quantum, are presented to us, their basic objects seem to be just spacetime points. All else - matter, fields, and even the metrical structure of spacetime, the 'chronogeometry' of the world - are represented as mathematical structures defined on these points; in particular, as just emphasised, physical quantities for matter and fields are represented by structures defined point by point. And so it is natural to presume that all these are to be construed as properties of (and relations between) points, or higher-order properties and relations. In short, we arrive at the doctrine now called 'substantivalism': that spacetime points are genuine objects, indeed are the basic objects of physical theory.

So much the worse, it seems, for the relationist tradition, stemming from Leibniz through Berkeley and Mach, of denying that space, time (and spacetime), and their parts, are objects; and of conceiving them instead as a system of spatiotemporal relations between bodies. For the bodies have become diaphanous and omnipresent fields, which are themselves complicated structures of properties and relations among spacetime points.

But we think one should be wary, both of this kind of argument for substantivalism, and of the doctrine itself. Space permits only the briefest statement of our reasons: we will mention two.

The first applies to any theory, such as general relativity, that is diffeomorphism invariant; or in the terminology of Section 4.1, to any theory whose only fixed structure (in the third sense of 'the same in all models') is the local topological and differential structure of a manifold - so that, intuitively, any map preserving this structure (i.e., any diffeomorphism) preserves the content of the theory. As mentioned at the end of Section 4.1, diffeomorphism invariance is widely taken to indicate that spacetime points are indeed not objects: their appearing to be so is an artefact of how we formulate the theory. This reason exemplifies a general point about objects or ontology. Namely: we should be wary of taking as the basic objects of our ontology (according to some theory) those items that are postulated as the initial elements in a mathematical presentation of the theory. For it might be just a happenstance of our formulation of the theory that these objects 'come first': a happenstance avoided by another formulation that can be agreed, or 
at least argued, to be better.

The second reason applies to any theory of physical geometry (where 'geometry' can be taken as including time as well as space, as in 'chronogeometry'). So it makes no presuppositions about what structure is fixed in the sense of Section 4.1. It has as its target, not the doctrine that spacetime points are 'real' but rather the doctrine that the metric structure of spacetime is intrinsic to it. In short: even supposing that spacetime points (or regions) are real, the fact that our only access, even in principle, to the metric structure of spacetime is through the behaviour (perhaps ideal behaviour) of matter - the proverbial rods and clocks - suggests that metric structure is not intrinsic to spacetime, but rather a relational matter reflecting the nature both of spacetime and of matter.

This last suggestion is very vague, but we cannot here develop it. It must suffice to mention one well-explored way of doing so, namely the Machian theories of Barbour et al. (For more details, see Barbour's article in the present volume, especially Section 4.) In a nutshell: these theories postulate

1. A space, often a relational one, i.e., a space whose geometry is fixed by the spatial relations between bodies;

2. A 'least-action' principle on this space, which defines both the dynamics of the system and the metric of time.

Incidentally, this definition of the temporal metric exemplifies the themes of Section 2 and 4.1. For this metric is emergent in the sense that it is not a basic postulate of the theory, and is also unfixed in the second and third senses of Section 4.1. But it is reduced in the same strong sense as the microcanonical measure in Section 2.2.2: i.e., it is explicitly definable in a language sufficiently rich to express calculus.

To conclude: we think these two reasons should make one cautious about any quantum gravity programme that postulates a spacetime manifold (whether fixed or unfixed, in the senses of Section 4.1) that, according to the theory, can be 'bare' (matter-free). And there are several such programmes, including the several approaches to quantum gravity based on the ideas of canonical quantization. 


\subsection{Interpreting Quantum Theory}

We turn to the last piece of 'stage-setting' needed for Section 5: issues concerning the interpretation of quantum theory. This is a large and complex subject, but fortunately our discussion can be very selective. Our topic of the emergence of time means that we must be concerned primarily with whether quantum theory has, or could have, the wherewithal to describe the classical spatio-temporal realm around us. Therefore we must take notice of the measurement problem, with its implicit threat that physical quantities pertaining to macroscopic objects take no definite value (but only a probability distribution) - contrary to our experience. More generally, we must be concerned with the interpretation of the quantum state-vector. But there are many interpretative issues about quantum theory, such as entanglement and non-locality, which we need not engage here .18

In fact, we need to raise two issues. The first arises in elementary quantum theory; the second has some aspects that are specific to quantum gravity. They are, in order:

(1) classical limits, approximations and decoherence in quantum theory;

(2) the interpretation of the quantum state; especially the quantum state of the Universe (which will be taken in the programmes focussed on in Section 5 as a functional of 3-geometries).

\subsubsection{Classical limits, approximations and decoherence in quan- tum theory}

One of course expects the emergence of time (with 'time' taken in our usual sense of 'classical spacetime') within a quantum gravity programme to involve some sort (or sorts) of classical limit of quantum theory, and/or some sort of classical approximation methods within quantum theory. And so it does, in our chosen quantum gravity programmes, as we shall see in Section 5 . Indeed, we will see there examples of each of the variety of approximations discussed in Section 2.4: (a) the neglect of some quantities; (b) the selection

\footnotetext{
${ }^{18}$ Another sense of 'describing the (unique) classical realm' will occur in the discussion in Section 5.5.4 of the no-boundary proposal: a sense related to the idea of a theoretically motivated boundary condition.
} 
of a subset of states; (c) combinations of (a) and (b), i.e., a subset of states approximating another theory as regards some quantities.

Here we just register that these various ways also occur even in the context of elementary quantum theory; and that the ideas and techniques used in this context do get applied (often suitably adapted) by the quantum gravity programmes discussed in Section 5.

- As to (a): we said in Section 2.4 that it was well-nigh universal practice in physical theorizing. In elementary quantum theory, (a) is also appealed to in foundational arguments. And this occurs in various ways. First, various approaches to the measurement problem argue that one need only avoid macroscopic indefiniteness of value, for a select subset of quantities. Second, in the theory of decoherence (more details below), neglecting some quantities (roughly, quantities defined on the environment of the system one is concerned with) is the crucial point in a physical argument that one avoids macroscopic indefiniteness of value for some other quantities (defined on the system).

- As to (b): when a probabilistic theory such as quantum mechanics replaces a deterministic one such as classical mechanics, one naturally hopes that the expectation values of some quantities in the probabilistic theory might obey the same equation of motion as the actual values obeyed in the earlier theory. In general this is not so for quantum mechanics; even for the basic quantity of classical equations of motion, viz. position. But there are circumstances in which it is so, or approximately so, for some special states.

- As to (c): In some cases, expectation values for quantities such as position, approximately obey the classical equation, because special conditions relating both to the states involved, and to a selection of quantities, hold good.

We emphasise that, for the most part, these ideas and techniques about classical limits and approximations are not controversial. That is: they can be invoked in order to address the measurement problem, or more generally issues about the interpretation of the state-vector; but they do not in themselves solve such problems - they at most contribute to (the technical core 
of) a solution. In particular, this applies to the use of these ideas and techniques in quantum gravity: so nothing in the use made of them in Section 5 will count as solving these problems!

Similar remarks apply to another idea: decoherence. That is to say: decoherence is an important aspect of quantum theory's 'recovery' of a classical realm; it has been well-explored in the context of elementary quantum theory, and has been invoked in the quantum gravity programmes in Section 5 . But we stress that it is not itself a solution to the measurement problem.

Here we just need to register the main idea. Roughly speaking, decoherence is the physical process of the diffusion of coherence from a system to its environment; here, 'coherence' refers to the characteristic interference terms of a quantum superposition, that distinguish the superposition from a classically interpretable mixture of states. In a bit more detail: decoherence is a process that rapidly puts macroscopic objects (including tiny ones such as dust particles) that interact with their environment (even very slightly, such as with the microwave background radiation) into mixtures, whose components are, in typical cases, approximate position eigenstates. The process diffuses the interference terms characteristic of the macro-object's initial superposition into the environment, so that its statistical behaviour is as if it is in a mixture - and a mixture of states that have definite values for familiar quantities like position, or quantities very 'close' to those like position. But we stress that this mixture is 'improper', i.e., is not interpretable as a matter of ignorance of which component is possessed. For a recent review, cf. [18.

\subsubsection{The meaning of a quantum state of the Universe}

But we also need to say something about the interpretation of the quantum state. This is not just a matter of it always being salutary to emphasise the vulnerability of quantum theory! Though most quantum gravity programmes put more pressure on the formalism of general relativity, than on the basic formalism of quantum theory, the endeavour of quantum gravity does put pressure on the interpretation of quantum theory - at least in the sense of showing how debatable most interpretative tenets are! We will make two main points. The first is specific to quantum gravity; the second is more general, but will also be needed in Section 5 .

\footnotetext{
${ }^{19}$ This pressure seems to us at least equal to the interpretative pressure that quantum gravity put on general relativity; for example, the trouble it cause for substantivalism.
} 
(1) First, the endeavour of quantum gravity is closely related to quantum cosmology, i.e., the attempt to have a quantum theory of the whole universe. And in quantum cosmology, the traditional Copenhagen interpretation, with its requirement of an observer external to the system, is obviously inapplicable.

A related point is that among the variants of the Copenhagen interpretation is one that requires, not an observer, but a 'classical background' external to the system. And, arguably, one obvious candidate for such a background is the continuous spacetime manifold of our usual quantum theories (both quantum mechanics and quantum field theories). But we admit to giving this sort of interpretation little credence. For we see no good argument for the necessity of such a background manifold as, for example, (in Bohr's words) a 'precondition of unambiguous communication'. एव

(2) In general, the difficulty of interpreting the quantum state makes it tempting to say something like (' $\mathrm{N}$ ' for naive):

(N): $\int_{\Delta}|\Psi|^{2}$ is the probability for the the values of a quantity $A$ to be in $\Delta$

where $\Delta$ is a subset of the spectrum of the self-adjoint operator $\hat{A}$ (representing the physical quantity $A$ ) to which the spectral theorem has been applied to obtain a representation of vectors in the Hilbert space as functions on $\hat{A}$ 's spectrum.

Indeed, it seems that $(\mathrm{N})$ states the 'core' of the Born interpretation of the quantum state; and yet avoids appealing to measurement, and also avoids (i) extra values for quantities beyond the orthodox eigenvalue-eigenstate link ('hidden variables'), and (ii) a physical collapse of the quantum state. This temptation to assert the proposition $(\mathrm{N})$ is not specific to quantum gravity or quantum cosmology: $\Psi$ need not be the state of the universe.

But of course, (N) is hopelessly vague. First, it is clear that if the quantum state does not collapse into having its support confined to $\Delta$ (as (ii) requires), then the phrase 'the values ...to be in $\Delta$ ' commits one to the extra values vetoed by (i). For what can the phrase mean except that the quantity concerned takes a value in $\Delta$ even though the support of $\Psi$ is not confined to $\Delta$ ?

\footnotetext{
${ }^{20} \mathrm{Cf}$. the rejection in Section 3.4 of Kantian requirement for a manifold. No coincidence of course, given the tenability of a Kantian reading of Bohr.
} 
And once we accept that there are some such extra values, we face various well-known hard questions.

1. Is there a preferred quantity or quantities $A$ which get such values (rather than all quantities as suggested by the formal schema $(\mathrm{N})$ )?

2. If so, can we choose the quantity or quantities so as to help with the measurement problem, i.e., so as to secure a description of a classical realm?

3. There are also formal questions. Do the quantities and values chosen avoid the traditional no-hidden-variable theorems: both the algebraic theorems in the tradition of von Neumann, and the non-locality theorems in the tradition of Bell? And how do the values change over time? That is: if we accept such values, we are obliged to give a rule specifying how they evolve (deterministically or stochastically) over time. And this rule must of course mesh with our answers to questions 1 . and 2. In particular, if in answer to question 2. we secured a classical realm at an instant, the rule for the evolution of values should not lose it later!

We need not consider how the various interpretations of quantum theory (such as the pilot-wave and modal interpretations) address these questions. But we should briefly discuss the Everettian interpretation, since it has been considered attractive in interpretative (and, even more, in popular) discussions of quantum gravity.

The basic Everettian idea is familiar from the philosophy of elementary quantum theory. It is that the particular classical realm that is apparent to us corresponds to just one component (summand) of the universal statevector - which always evolves deterministically, never collapsing. As to the above questions, Everettians traditionally tended to answer questions 1. and 2 . by proposing as the 'preferred basis' in terms of which to resolve the universal state-vector, the approximate position eigenstates (i.e., narrowly peaked wave-functions) of measurement pointers: a proposal that was widely, and we think rightly, criticized as giving too fundamental a role to the notion of measurement; (for example, [19]). But in recent years, they have instead invoked the ubiquitous and very efficient process of decoherence to give a principled, dynamically motivated, specification of the preferred basis. We 
think this is an improvement. In particular, it helps an Everettian explain why the other classical realms he or she postulates as corresponding to the other components of the universal state-vector remain 'hidden' from us: decoherence makes the interference terms, that would reveal these realms, quickly become far too small to be detected. थ

As to question 3. above, Everettians traditionally tended not to give a rule of evolution (even a stochastic one) for the values of their preferred quantities. But recently they have done so, often by adopting the 'consistent histories' formula for multi-time probabilities ${ }^{22}$

So much for the general problems of $(\mathrm{N})$. If we apply $(\mathrm{N})$ to quantum geometrodynamics, in which $\Psi$ is a wave-functional on 3-geometries, we get yet more interpretative problems. We explain these in Section 5, especially Section 5.3.

\section{Quantum Geometrodynamics}

\subsection{Prospectus; the Problem of Time}

So much by way of preliminaries! In the rest of this paper, we will discuss how the idea of the 'emergence' of time appears in one particular approach to quantum gravity: namely the canonical quantization programme, in the form that leads to the famous Wheeler-DeWitt equation.

We shall discuss the emergence of time in two main contexts, the first acting as a prerequisite of the second. ${ }^{35}$ The first, discussed in Sections 5.25.4 , is the Wheeler-DeWitt equation per se. Here, the canonical quantisation of general relativity leads to a quantum state-vector which is a functional of 3-geometries; (this subject is known as 'quantum geometrodynamics'). But there are immense mathematical and conceptual difficulties about this quantisation. Prominent among the conceptual difficulties is what is what is perhaps the best-known philosophical topic arising from quantum gravity: the so-called 'problem of time', discussed in detail in this volume by Kuchar. ${ }^{4}$

\footnotetext{
${ }^{21}$ For more discussion see, for example, 20 Section VI, and 21 Sections 2, 3.

${ }^{22}$ For more discussion see, for example, 21] Section 5, 6 .

${ }^{23}$ For both contexts, we will be concerned mainly with the emergence of local, rather than global, aspects of space and time. For discussion in this volume of the global structure of time, see the essays by Torretti and Lucas.

${ }^{24}$ We heartily recommend Kuchař, this volume, as a non-technical review. Among the
} 
We also will emphasise the problem of time, since as we shall see it is closely linked to the emergence of time.

In order to discuss the emergence of time in the face of these various difficulties, our strategy needs must be, after describing such a difficulty, to set it aside - if only to raise another! More precisely, our plan, as regards this first context, will be:

1. To introduce the problem of time in very general terms (in this Subsection).

2. To introduce the canonical quantisation of general relativity. This will include discussing (i) the origin and status of the Wheeler-DeWitt equation; and (ii) the more specific forms of the problem of time, and their relevance to the emergence of time (Section 5.2).

3. To discuss the problem of interpreting a solution of the Wheeler-DeWitt equation, supposing we could somehow be given one (Section 5.3).

4. To discuss the idea, which arises from some of the material in Sections 5.2 and 5.3, that time is an approximate and state-dependent concept pertaining to a semiclassical approximation to quantum geometrodynamics.

In Section 5.5, we will turn to our second main context: the so-called 'Euclidean' programme, that aspires to produce a particular solution of the Wheeler-DeWitt equation using a functional-integral over (Riemannian) metrics on a four-dimensional space. The goal is usually a theory of quantum cosmogenesis - i.e., a theory that predicts a single, unique state for the quantum universe.

The material in Sections 5.2-5.4 will be a prerequisite for this discussion. Indeed, there are two main connections here. First, as we mentioned in Section 1: the Euclidean programme's treatment of the emergence of time is beset by the problem of time; and one of our goals is to correct the impression in the popular literature about the Euclidean programme (especially about the Hartle-Hawking no-boundary proposal) that one can discuss the emergence of time without having to address the problem of time. Second,

more technical reviews are [22, 23]; see also [1]. Our paper 111] also discusses some other aspects of quantum gravity from a philosophical perspective. 
more technically: the treatment of the emergence of time in the Euclidean programme relies on the treatment in Section 5.4 in terms of semiclassical approximations.

What then is the problem of time? As emphasized by Kuchař, it is really a cluster of problems that arise principally from the disparate ways in which time is treated in quantum theory and in general relativity. The main contrast goes back to the notion of fixity that we discussed in Section 4.1: in quantum theory, time is treated as a part of the fixed, theoretical background structure, in all three of senses in Section 4.1 classicality, nondynamicalness, and being the same in all models.

On the other hand, in general relativity - which is, after all, a unified theory of space, time and gravity - time is only fixed in the first sense of being classical. It is not fixed in the third sense in so far as, for example, what counts as a timelike vector depends on the space-time geometry, and is hence model-dependent. And since this geometry is the subject of dynamical laws, we can also say that time is not fixed in the second sense. In short, time is treated as an aspect of the system. Various conceptual and technical difficulties in constructing a satisfactory theory of quantum gravity can be traced, at least in part, to this underlying difference in the 'ingredient' theories.

The problem of time has been most studied in geometrodynamics, i.e., for the canonical (Hamiltonian) approach to quantum gravity, using standard geometric variables. Accordingly, Kuchař (this volume) confines his discussion to this approach, and we shall follow him in this. But we note that the problem of time takes on a very different appearance (and is generally less studied as such) in other approaches to quantum gravity such as, for example, the superstring programme.

Finally, by way of prospectus: we should add that Kuchař distinguishes three main forms of the problem of time (as it appears in geometrodynamics), discussing them in order. We will describe them in Section 5.2. But in short, they are:

(i) the difficulty of finding an 'internal time' in classical general relativity;

(ii) the difficulty of finding a time 'buried' in the Wheeler-DeWitt equation; and

(iii) the difficulty of interpreting the Wheeler-DeWitt equation in a fundamentally timeless way, and accordingly treating time within our 
present-day theories, general relativity and quantum theory, as an approximate concept.

Our discussion in Section 5.2 will follow the same order as Kŭchar; but since our topic is the emergence of time, we will of course emphasise (iii).

\subsection{The Canonical Quantisation of General Relativity}

\subsubsection{Two types of canonical quantisation}

As we said in Section 3.3, the basic strategy of quantum geometrodynamics is to cast general relativity in Hamiltonian form, so that it describes the evolution in time of the geometry of a 3 -dimensional spacelike hypersurface, $\Sigma$; and then to quantize this Hamiltonian theory.

First, we should distinguish two different procedures, or 'recipes', for quantizing a Hamiltonian theory. Both are called 'canonical quantization', but the first (dating from the 1920s) suits a Hamiltonian theory in which the number of variables in the formalism equals the number of physical degrees of freedom of the system described. For example, the classical Hamiltonian mechanics of a point particle moving in one dimension with position $x$, is quantized by identifying a quantum state as a complex-valued function of position $\psi(x)$, which is to evolve in time according to the Schrödinger equation (so that we write $\psi(x, t)$; or, better, $\psi_{t}(x)$ ): more generally, any physical variable $A$ becomes a linear operator $\hat{A}$ that acts on the quantum states $\psi$.

The second type of canonical quantization (dating from the 1950s) suits a Hamiltonian theory in which there are more variables in the formalism than there are physical degrees of freedom; accordingly, the theory contains extra equations that relate some variables to others. These equations are called 'constraints', and the theory (or the system) is called 'constrained'. One strategy for quantizing such a system is to use the constraints to eliminate some variables (this is called 'solving the constraints'), so as to get an unconstrained theory, which is then quantized using the first procedure above. But an alternative procedure is to quantize without solving the constraints. Broadly speaking the idea is that given a classical constraint equation, $c=0$ say, where $c$ is some function of the variables, one requires that the quantum state $\psi$ obey $\hat{c} \psi=0$, where the 'hat' indicates that the variables in $\hat{c}$ have become operators. This is called 'constrained quantization'

\footnotetext{
${ }^{25}$ Strictly speaking, this procedure is only correct for what are called 'first-class' con-
} 


\subsubsection{The Wheeler-DeWitt Equation; its origin and status}

It transpires that classical general relativity in Hamiltonian form is a constrained system; indeed the dynamics of the theory is coded in the constraints (Kuchař's 'teorema egregium', equation (5) of his paper in this volume). So one strategy for quantizing the theory is to try to solve the constraints before quantizing. This turns out to involve trying to find a so-called 'internal time' as a function of the canonical variables of classical general relativity: a time which could then serve as a time for the Schrödinger equation of the quantized theory. This is the most conservative strategy from the quantum perspective since it manipulates classical general relativity to get the kind of time that is used in ordinary quantum theory. Unfortunately, however, this cannot be done by simply taking over one of the familiar 'ordinary' times of classical general relativity (for example, local proper time, or the global time variables characteristic of certain simple cosmological models). Rather, one has to confront the extremely difficult problem of solving a collection of very non-linear, coupled (elliptic) partial-differential equations. So far, this has proved intractable. This, then, is the first form of the problem of time: the problem of finding a time 'before quantisation'.

Another strategy is to try constrained quantization. Thus one tries to quantize the Hamiltonian form of general relativity, by postulating a complex-valued function of 3-geometry ${ }^{20} \Psi[h]$ which will be subject to appropriate operator constraints of the form $\hat{C} \Psi=0$. Note that, by writing our prospective quantum states as just functionals on superspace $\Psi[h]$, we have ignored matter. To allow for matter fields, $\phi$ say, we need to write a quantum state as $\Psi[h, \phi]$. However, for simplicity we will ignore this qualification

straints. We should also stress that there are other procedures for quantizing a classical theory, the best known of which - called 'path-integral' quantization - was developed principally by Feynman. It is more naturally adapted to a Lagrangian, rather than Hamiltonian, form of a classical dynamics; we shall return to it in Section 5.5.

${ }^{26}$ The square bracket indicates that the argument is itself a function (namely a specification of a 3-geometry on an entire spacelike slice). Such a 'function of a function' is usually called (by physicists) a 'functional'. The set of all 3-geometries (on a given 3-manifold $\Sigma$ ) is called 'superspace'. It is a infinite-dimensional space, since two 3-geometries can differ from one another in infinitely many (independent) ways. Sometimes, the word 'superspace' is used for the quotient set obtained by factoring out the action of the spatial diffeomorphisms, associated with momentum constraint discussed below. This quotient space is also infinite-dimensional. 
in what follows, writing just $\Psi[h]$ etc-despite our sympathy, expressed in Section 4.2, for the idea that matter is needed to make sense of geometry.

It transpires that general relativity has two types of constraint, called the 'momentum' and the 'Hamiltonian' constraints. When we quantize by requiring the 'hatted constraints' to send any state $\Psi[h]$ to 0 , we find that the momentum constraint is readily interpreted: it requires that $\Psi$ should take the same value at any pair of 3 -geometries $h, h^{\prime}$ that differ only by a (smooth) permutation of the points of the 3 -dimensional space $\Sigma$ on which they are defined. That sounds reasonable since $h, h^{\prime}$ seem physically equivalent, the points just functioning as 'pegs' on which to 'hang' the fields.²]

But the quantum form of the Hamiltonian constraint is very obscure. In the context of geometrodynamics, it is the famous Wheeler-DeWitt equation which, for the sake of reference, we give here as

$$
\begin{array}{r}
-\frac{\hbar^{2} \kappa^{2}}{2}(\operatorname{det} h)^{-1 / 2}(x)\left(h_{a c}(x) h_{b d}(x)+h_{b c}(x) h_{a d}(x)-h_{a b}(x) h_{c d}(x)\right) \frac{\delta^{2} \Psi[h]}{\delta h_{a b}(x) \delta h_{c d}(x)}- \\
\frac{(\operatorname{det} h)^{1 / 2}(x)}{\kappa^{2}} R^{(3)}(x) \Psi[h]=0
\end{array}
$$

where $\kappa^{2}:=8 \pi G / c^{2}$, and $R^{(3)}(x)$ is the curvature scalar formed from the 3-metric $h$.

From the classical analogue, one would expect this equation to express the dynamical content of the theory. Thus one might expect $\Psi[h]$ to evolve according to some sort of Schrödinger equation. But the Wheeler-DeWitt equation (5.1) contains no explicit time parameter, so that to make sense of the equation as describing evolution, one apparently needs to find a time 'buried' in it and its associated formalism. And here we meet the problem of time in its second form: it is very difficult to find such a time 'after quantisation'. This is the primary form taken by the problem of time in geometrodynamics.

The difficulty of finding a buried time in the Wheeler-DeWitt equation (and the related difficulty of finding an 'internal time' before quantisation)

\footnotetext{
${ }^{27}$ This is one of several points where the problem of time is related to the diffeomorphism invariance of general relativity, and Einstein's 'hole argument'. As discussed in Section 4.1, diffeomorphism invariance suggests that spacetime points should not be taken as real objects, even in interpreting classical general relativity. In the canonical approach to quantum gravity, this argument applies most immediately to points in space rather than spacetime.
} 
prompts the idea that geometrodynamics, and perhaps quantum theory in general, can - or even should - be understood in an essentially 'timeless' way. For if that were so, then one would not expect time to appear in the basic postulates of the theory: it would be an approximate concept, associated perhaps with (i) some states (solutions) but not others; or with (ii) some ranges of some variables but not other ranges. This then is the third form of the problem of time: to identify such an approximate concept, and argue that it is adequate. Thus we arrive at our own topic: the emergence of time.

Note however that the second and third forms of the problem of timefinding a time 'buried' in the Wheeler-DeWitt equation, and finding some approximate notion of time in a timeless geometrodynamics - should not be sharply distinguished. In particular, the line of work we shall discuss in Section 5.4 -5.5 - the use of ideas and techniques about semi-classical approximations drawn from usual quantum theories - could arguably come under either heading, since it involves extracting an approximate notion of time from the Wheeler-DeWitt equation.

But before discussing this line of work (in Sections 5.4 and 5.5), we must sound a note of warning. We must emphasize the extreme interpretative difficulties that surround not only this line of work, but also all attempts to interpret solutions of the Wheeler-DeWitt equation. Indeed, there are two points to be made here. First, the difficulties of interpreting the quantum state of the universe, discussed in Section 4.3.2, persist; and they become entangled with the problem of time (Section 5.3).

Second, much of the discussion that follows is generically deficient in the sense that no one who properly studies the mathematics that lies behind the Wheeler-DeWitt equation could seriously contemplate that the equation has non-trivial solutions in any normal sense (other than in gross approximations to the equation, such as that given by using minisuperspace from one perspective, the notorious problem of the non-renormalisability of perturbative quantum gravity rears its ugly head again at this point. True: the main hope of the Ashtekar approach is that it may eventually yield gen-

\footnotetext{
${ }^{28}$ Models that take account of only a finite number of the dimensions of superspace are called 'minisuperspace' models. Though this sort of restriction is radical, it need not involve neglecting all but a tiny spatial patch of the 3-geometry; for a sufficiently homogeneous 3-geometry might be specified by a finite number of numbers. The usual, simplest example is where the entire 3-geometry is coded by a single number: the radius of a perfectly homogeneous and isotropic universe.
} 
uine solutions to its own analogue of the Wheeler-DeWitt equation. However, this analogue involve functions of spin-connections, or loops in 3-space, and hence the interpretation would be quite different from that of geometrodynamics.

This situation inevitably prompts the sceptical question why we are spending time and effort discussing possible philosophical implications of an equation that is mathematically meaningless! Are we as misguided as the medieval scholastics are often taken to have been, in their discussions of how many angels can dance on the head of a pin? We believe not. Indeed, as philosophers know well, the scholastics' discussions were not as misdirected as folklore suggests. They addressed deep, maybe perennial, issues about personal identity and spatiotemporal location, in terms of their era's accepted ontology; which included angels. We would like to think that our discussion stands up as well. ${ }^{29}$

\subsubsection{The problem of time vs. the emergence of time}

At this point we should emphasise that although the problem of time and the emergence of time are closely connected in various ways, they are distinct topics; even within geometrodynamics. There are two main differences. 50

Perhaps the most obvious difference is that the problem of time is mostly about time rather than space; while as we said in Section 1, we intend 'the emergence of time' to also cover the emergence of spacetime, and so space. At a technical level, the tendency not to dwell on a 'problem of space' stems from the fact that, as noted above, in constrained quantization, the momentum constraint — which, from a spacetime perspective, shuffles spacetime points within a given 3-dimensional spacelike slice - is much easier to deal with, conceptually and technically, than the Hamiltonian constraint, which is related, at least classically, to diffeomorphisms that map points from one spacelike slice to another.

The second difference arises from the fact that the emergence of time is about the emergence of classical spacetime, simply because all our present

\footnotetext{
${ }^{29}$ Or at least, stands up as well to its own era's scrutiny: as one might say - for them, the Spanish Inquisition; for us, the Research Assessment Exercise!

${ }^{30}$ The difference is clearer if we go beyond geometrodynamics: Section 3 makes it clear that the emergence of time can be discussed apart from geometrodynamics - to which our formulations of the problem of time are clearly confined.
} 
theories - whether classical general relativity or a quantum theory of forces other than gravity - treat spacetime classically. On the other hand, the problem of time is about 'finding' a time in quantum gravity theories: and so not, in the first instance, about finding some kind of classical limit of, or classical approximation to, such theories. (A qualification: the third form of the problem of time, above, is often focussed on finding such a limit or approximation.) So the emergence of time is more directly connected to questions about the classical limit (and, more generally, interpretation) of quantum theory, than is the problem of time (except in its third form). We shall see this sort of connection in more detail in Section 5.4 .

\section{$5.3 \quad$ Interpreting $\Psi$}

Suppose we are given a solution to the Wheeler-DeWitt: a functional $\Psi$ of 3 -geometries. How should it be interpreted?

First of all, one must beware of a tempting error about the relation between superspace and Lorentzian 4-manifolds (spacetimes). It is tempting to think that a curve in superspace determines a spacetime; and thus in cases where Einstein's equations are satisfied, a model of general relativity. But in general, this is not so: a curve in superspace does not determine a spacetime, since the curve does not dictate how its points, the 3-geometries, are embedded in the spacetime. Technically, the latter information is coded in the 'lapse function' and the 'shift vector': if these are given, then a spacetime is determined. Conversely, a spacetime can be foliated in many ways into a 1-parameter family of 3-dimensional slices each with a 3-geometry. So in this sense, certain type of sheaf of curves is equivalent to a spacetime. Note that, in some simple (minisuperspace) models which neglect all but a finite number of the infinite dimensions of superspace, the lapse and shift are in effect fixed, and in this case a curve in superspace does determines a spacetime.

With this warning in hand, let us press the question: How should we interpret $\Psi$ ? One possibility might be to say that $\Psi$ 'predicts' a spacetime with a particular 4-geometry $g$ if (i) it has a non-zero value on any 3-geometry obtained by restricting $g$ to a spacelike hypersurface of the spacetime; and (ii) any 3 -geometry $h$ such that $\Psi[h] \neq 0$ arises as the metric induced by $g$ on some 3-dimensional spacelike hypersurface.

However, such a view wholly neglects the way in which a wave-function is used in standard quantum-theory to predict probabilities. With a view 
to recovering the Born-rule, it is tempting to return to the interpretation labelled $(\mathrm{N})$ at the end of Section 4.3, but now applied to superspace. That is, it is tempting to say that

$(\mathrm{N}): \int_{\Delta}|\Psi[h]|^{2} \mathcal{D} h$ is the probability for the 3 -geometry $h$ to 'be' in the set $\Delta$.

This has been called the naive Schrödinger interpretation 24]: hence the 'N'; but what are we to make of it? Suppose we set aside all technical worries about the definition of the measure symbolized by $\mathcal{D} h$. Suppose also we set aside general worries about the meaning of (presumably objective) probability for a state of the whole universe. 15 Still there is trouble, because we expect theories to talk of probabilities of events at a given time, while $\Psi$ and $(\mathrm{N})$ make no reference to time.

To recover a time in the context of $(\mathrm{N})$, it is natural to hope that $\Psi$ will take a form that 'contains' a time. The obvious idea is that - setting aside the point above that a curve in superspace does not uniquely determine a spacetime $\Psi$ should take non-negligible values only on a single curve in superspace. If so, one might think of $\Psi$ as 'predicting' the corresponding spacetime. Or, perhaps more realistically, one might hope that $\Psi$ takes nonnegligible values only on a 'strip' around a curve in superspace; in which case one might hope to identify some ensemble of curves all lying in this strip, and even to use the values of $|\Psi[h]|^{2}$ at various points in the strip to talk about the probability of evolving from one point to the other. And one might hope to make out some such interpretation in the more general case where $\Psi$ takes non-negligible values on a criss-crossing set of strips.

But the trouble with schemes of this type is that there is no reason to believe that, even if they existed, solutions to the Wheeler-DeWitt equation would have anything like these properties. Also, we must emphasise that the qualification "if they existed" should be taken very seriously: we recall our earlier caveats about the mathematically ill-defined nature of the WheelerDeWitt equation.

In addition to this particular reservation, a simple variable-counting argument shows that the sense in which $\Psi$ 'contains' a time is quite different from, and more subtle than, the above ideas about parameterizing curves

\footnotetext{
${ }^{31}$ Some modal metaphysicians are happy enough to talk of such probabilities; for example, 25.
} 
in superspace. In essence, it is a matter of time being a degree of freedom (at every point in the 3-manifold $\Sigma$ ) within the argument, $h$, of $\Psi$. More precisely: to specify a 3-geometry on the space $\Sigma$ requires 6 real numbers at every point of $\Sigma$; three of these correspond to making a choice of the otherwise arbitrary coordinates on the 3 -space (this is closely related to the spatial diffeomorphisms associated with the momentum constraint), and two correspond to the physical degrees of freedom of the gravitational field (the two circular-polarization modes of a weak gravitational wave) - but the last corresponds to the 'internal time' ('time before quantization'), mentioned at the start of Section 5.2.2. This means, incidentally, that in seeking a time within $\Psi$ (in accordance with the 'time after quantization' strategy), one is in fact thrown back to the same kind of technical and conceptual problems that beset the strategy of seeking a time before quantizing. In short, we are back in murky waters!

\subsection{Semiclassical Approximations in Quantum Geometro- dynamics}

We shall now discuss the emergence of time in quantum geometrodynamics in the approach that aspires to find time as an approximate, semi-classical concept that arises 'after quantization'. (Since, as remarked above, time does not emerge in the other main form of quantum geometrodynamics ('time before quantization'), this restriction is not as limiting as it might seem.)

The main idea is that (i) time should only emerge in the context of some special states that are approximate semiclassical solutions to the WheelerDeWitt equation; and (ii) the time-variable that emerges depends on the state chosen.

More precisely, one begins by considering a state of the form $\Psi[h]=$ $A[h] \exp (i S[h])$, subject to the conditions that $S$ and $A$ are real, and $A$ is 'slowly varying' in comparison with $S$. Or, allowing for matter, one begins with a similar form that incorporates matter variables $\phi$. This ansatz is then substituted into the Wheeler-DeWitt equation, and one seeks a solution as a power-series in $S[h]$ (the so-called WKB expansion). The leading-order contribution has the following significant properties:

(i) The exponent $S[h]$ obeys the same equation as does a classical quantity (the 'action') in general relativity (viz. the Hamilton-Jacobi equation). 
Furthermore, this connection between the phase of the quantum state and the classical action is parallel to similar connections established in standard quantum theories.

(ii) If one can find a state-dependent (indeed, $S$-dependent) 'internal time', $\mathcal{T}$, obeying certain conditions, then the entire lowest-order contribution to $\Psi[h]$ (or allowing for matter, $\Psi[h, \phi]$ ), obeys a Schrödinger equation that uses a time-derivative with respect to this time $\mathcal{T}$.

The general character of the problems faced by this approach can be summarized as follows. First, since one starts with the Wheeler-DeWitt equation, one encounters the problems mentioned earlier: in particular, the extreme difficulty of giving the scheme even the semblance of a proper mathematical meaning. In addition, it is extremely difficult to find internal time variables except in the simplest models - where, for example, the internal time might be the radius of the universe. And, since the basic interpretation of $\Psi$ is obscure, it remains unclear how the fact that $S$ obeys the Hamilton-Jacobi equation of general relativity is meant to yield classical spacetimes.

But there are also problems that are distinctive of the semiclassical approach itself. Some of these relate to technicalities: for example, what should we make of the fact that when the next term in the WKB expansion is taken into account, the form of the Schrödinger equation is lost? But there are also purely conceptual problems. Among the most interesting, from the viewpoint of the emergence of time, is the question of how the semiclassical approach proposes to recover a description of the (apparently) unique classical spacetime around us, despite the facts that in this approach:

(i) a single WKB state-i.e., a single $S$-will correspond to a family of classical spacetimes, albeit all using the same internal time $\mathcal{T}$; (though much is obscure in the recovery of classical spacetimes, this one-many correspondence follows from a variable-counting argument, and is not particularly controversial);

(ii) there seems no reason to exclude superpositions of WKB states, each term in which gives rise to its own time $\mathcal{T}$ and its own family of spacetimes.

This last remark returns us to the topics of Everettian interpretations, and decoherence, discussed in Section 4.3. We shall not enter details about 
the first of these, which is much discussed. Let us just recall (from Section 4.3.2) that the Everettian denies that there is a sheer happenstance about which 'classical realm' is real, i.e., about which 'branch' of the universal state is realized. Rather, the Everettian proposes that all the realms ('branches') are realized. In the present discussion, the Everettian can presumably take a similar position. He or she can deny that there is sheer happenstance about which classical spacetime, among the family or families given by (i) or (ii), is realized. Rather, all the spacetimes are realized.

But we should briefly discuss how the ideas and techniques for modelling decoherence in usual quantum theories have been adapted to semiclassical quantum geometrodynamics. Recall (from Section 4.3.1) that decoherence is the diffusion of coherence: it is the process whereby for a system interacting with its environment, the interference terms characteristic of a coherent superposition very rapidly become far too small to be detected, leaving the system in an (improper) mixture of states that are definite for familiar quantities like position.

Of course, to adapt this notion to quantum geometrodynamics (at least, as applied in the context of quantum cosmology), one must allow for the fact that - if defined as the totality of all that is - the universe has no environment! In practice, studies of decoherence within quantum geometrodynamics use some of the variables in the model (either gravitational or matter variables) to act as an environment for the others: these others constitute the 'system' whose state one wants to show to be a mixture. Typically, inhomogeneous variables - i.e., roughly, spatially varying variables - are used as the environment of the homogeneous modes. Thus one argues that the apparent classical spacetime, with a spatially homogeneous geometry and matter-distribution (on a large scale), is recovered; at least in the sense that one obtains a mixture of states (albeit an improper one) that are each approximately definite for the homogeneous variables. So in particular, a superposition of two or more WKB states (as in (ii) above) that are defined only on the homogeneous modes will rapidly evolve, to a very high degree of approximation, to a corresponding mixture. And, as in Section 4.3.2, the Everettian who maintains that there is a classical spacetime for each such state, can appeal to decoherence to explain why the real spacetimes other than our own are 'hidden' from us: viz., decoherence makes the interference terms that would reveal them far too small to be detected.

But we should also note two problems concerning decoherence in semi- 
classical quantum geometrodynamics; (we set aside general doubts about the contribution of the idea of decoherence to solving the measurement problem in quantum theory, for example based on the fact that the mixture one obtains is improper). First, we should stress that the tracing out of the 'environment' variables to obtain a mixture is ill-defined for reasons that, though technical, are intimately tied to the conceptual problems about interpreting the Wheeler-DeWitt equation 26, 22].

The second problem is more general, and more basic. There is an obvious, major conceptual difference between decoherence, as understood in usual quantum theories, and as used here. In the former context, time is fixed in the senses of Section 4.1, and decoherence is a process that takes place in time. Here, on the other hand, there is meant to be no time at the fundamental level; but somehow, decoherence is meant to operate at this level to make time emerge. So whatever 'decoherence' may mean, it cannot be construed as a temporal process.

So much by way of reviewing the emergence of time in quantum geometrodynamics. We will see in the next Section how the problems described in this review - the various aspects of the problem of time, the murkiness of the interpretation of a functional $\Psi$ on superspace, and the problems of the semiclassical approach - all apply to the Euclidean programme's approach to canonical quantum gravity. Indeed, its treatment of the emergence of time relies on the treatment given by semiclassical quantum geometrodynamics.

\subsection{The Euclidean Functional Integral Approach to Canonical Quantum Gravity}

\subsubsection{The general idea}

The general idea is to construct wave-functionals on 3-geometries with the aid of a functional integral over manifolds endowed with a Euclidean metric. To this general idea, the no-boundary proposal adds a specification of which types of manifold one should consider.

The origin of the 'Euclidean' approach to quantum gravity lies in Hawking's remarkable discovery in 1974 that a black hole will radiate particles with a thermal spectrum via a quantum-mechanical process [27]. Hawking's results were quickly rederived using thermal Green's functions which - in normal quantum field theory - are closely connected with replacing time by an 
imaginary number whose value is inversely proportional to the temperature. This led Hawking to propose his 'Euclidean' quantum gravity programme in which the central role is played by Riemannian, rather than Lorentzian, metrics (this being the appropriate curved-space analogue of replacing time $t$ with $\sqrt{-1} t$ ). In particular, Hawking proposed to study functional integrals of the form

$$
Z(\mathcal{M}):=\int \mathcal{D} g e^{-\frac{1}{\hbar} \int_{\mathcal{M}}|\operatorname{det} g|^{1 / 2} R^{4}(g)}
$$

where the integral is over all Riemannian metrics $g$ on a four-manifold $\mathcal{M}$ (and $R^{4}(g)$ is the curvature of $g$, and det $g$ its determinant). The expression Eq. (5.3) generalizes naturally to include a type of 'quantum topology' in which each four-manifold $\mathcal{M}$ contributes with a weight $\chi(\mathcal{M})$ in an expression of the type

$$
Z:=\sum_{\mathcal{M}} \chi(\mathcal{M}) Z(\mathcal{M})
$$

It is not easy to give a rigorous mathematical meaning to these objects but, nevertheless, the idea has been extremely fertile. In particular, if applied to a manifold with a single 3-boundary $\Sigma$, the expression Eq. (5.4) corresponds to a functional $\Psi[h]$ if the functional integral is taken over all 4-metrics $g$ on $\mathcal{M}$ that induce the given 3-metric $h$ on $\Sigma$. Furthermore, the functional of $h$ thus defined satisfies (at least, in a heuristic way!) the Wheeler-DeWitt equation Eq. (5.1); thus we might write (allowing also for matter variables)

$$
\Psi\left[h, \phi_{0}, \Sigma\right]=\sum_{\mathcal{M}} \chi(\mathcal{M}) \int \mathcal{D} \phi \mathcal{D} g e^{-\frac{1}{\hbar} I(g, \phi, \mathcal{M})}
$$

This expression is the basis of the famous Hartle-Hawking [2] 'wavefunction of the universe' in quantum cosmology. It is known as the 'noboundary' proposal, since it is based on the idea that there is a sense in which the universe has no 'initial' boundary. To be precise: the no-boundary proposal requires that the sum in (5.5) should be over compact manifolds $\mathcal{M}$ which have the (connected) three-manifold $\Sigma$ as their only boundary; where $\Sigma$ is to be, as in Section 5.2 onwards, the 3-manifold that carries the 3 -geometries of quantum geometrodynamics.

We can now see the basis for the metaphor, much used in the popular literature, that according to the no-boundary proposal, the universe is created by 'tunnelling from nothing'. The word 'nothing' just reflects (very 
obscurely!) the idea that $\mathcal{M}$ has only $\Sigma$ as its boundary. The word 'tunnelling' refers to the facts that (i) moving from a Lorentzian to a Riemannian manifold corresponds, roughly, to moving from a time variable that is a real number to one that is purely imaginary (in the sense of complex numbers); and (ii) in normal quantum theory, a good approximation to the probability of tunnelling through a potential barrier, can be found by computing the action $I$ for a solution to the classical equations of motion with an imaginary time; the probability amplitude in question is then proportional to exp $-I / \hbar$. We shall return to this latter point shortly.

\subsubsection{The emergence of time in the Euclidean programme}

It is clear that, like quantum geometrodynamics, the functional integral approach makes fundamental use of a manifold. This means not just that it uses mathematical continua, such as the real numbers (to represent the values of coordinates, or physical quantities); it also postulates a 4-dimensional manifold $\mathcal{M}$ as an 'arena for physical events'. However, its treatment of this manifold is very different from the treatment of spacetime in general relativity in so far as it has a Euclidean, not Lorentzian metric (which, apart from anything else, makes the use of the word 'event' distinctly problematic). Also, we may wish to make a summation over different such manifolds, as indicated in Eq. (5.5). Finally, as we shall discuss below, it is in general necessary to consider complex metrics in the functional integral (so that the 'distance squared' between two spacetime points can be a complex number), whereas classical general relativity uses only real metrics.

Thus one might think that the manifold (or manifolds!) does not (do not) deserve the name 'spacetime'. But what is in a name?! Let us in any case now ask how spacetime as understood in present-day physics could emerge from the above use of Riemannian manifolds $\mathcal{M}$, perhaps taken together with other theoretical structures.

The main answer is what one would guess on the basis of the remarks at the end of Section 5.5.1 about semiclassical calculations of quantum amplitudes. That is: let us first set aside the deep problems about the interpretation of $\Psi$. Then the state-vector defined by Eq. (5.5) will predict a classical spacetime (more precisely: the correlations between position and momentum variables that are characteristic of classical spacetime), where it 
is well approximated ${ }^{\text {[2 }}$ by the semi-classical approximation to the functional integral. Here, 'the semi-classical approximation to the functional integral' (also called 'the saddle-point approximation') means evaluating the integral by summing the contributions given by the stationary points of the action.

In particular: if we choose to specify the boundary conditions using the no-boundary proposal, this means that we take only those saddle-points of the action as contributors (to the semi-classical approximation of the wave function) that correspond to solutions of the Einstein field equations on a compact manifold $\mathcal{M}$ with a single boundary $\Sigma$ and that induce the given values $h$ and $\phi_{0}$ on $\Sigma$.

In this way, the question of whether the wave function defined by the functional integral is well approximated by this semi-classical approximation (and thus whether it predicts classical spacetime) turns out to be a question of choosing a contour of integration $C$ in the space of complex spacetime metrics. For the approximation to be valid, we must be able to distort the contour $C$ into a steepest-descents contour that passes through one or more of these stationary points and elsewhere follows a contour along which $\left|e^{-I}\right|$ decreases as rapidly as possible away from these stationary points. The wave function is then given by:

$$
\Psi\left[h, \phi_{0}, \Sigma\right] \approx \sum_{p} e^{-I_{p} / \hbar}
$$

where $I_{p}$ are the stationary points of the action through which the contour passes, corresponding to classical solutions of the field equations satisfying the given boundary conditions. Although in general the integral defining the wave function will have many saddle-points, typically there is only a small number of saddle-points making the dominant contribution to the path integral: (more precisely, that is what happens in normal quantum theory where the mathematical expressions are reasonably well defined).

For generic boundary conditions, no real Euclidean solutions to the classical Einstein field equations exist. Instead we have complex classical solutions, with a complex action. This accords with the account in Section 5.4 of the emergence of time via the semiclassical limit in quantum geometrodynamics,

\footnotetext{
${ }^{32}$ Strictly speaking, this is what is true of normal quantum theory. However, the possibility of making any proper mathematical sense of Eq. (5.5) - and hence of having a genuine approximation to something that genuinely exists mathematically - is extremely remote.
} 
in two senses: the first rather negative (more precisely: 'merely permissive'), the second more positive.

1. If the saddle-points of the integral did correspond to real solutions of the Einstein field equations, with real action $I$, the wave function would be of the exponential form $e^{-I / \hbar}$ (note that the sign of $I$ is not fixed). However, in order to predict classical spacetime, a solution of the Wheeler-DeWitt equation has to be of the form $e^{i S / \hbar}$, with the phase $S$ satisfying the Lorentzian Hamilton-Jacobi equation for general relativity. So, from that perspective, it is just as well that the saddlepoints give complex classical solutions, with complex action: it means there is some hope for recovering a classical spacetime.

2. More positively, one can show - at least, heuristically - that a WKB expansion of a state $\Psi$ arising from complex classical solutions (saddle points) with complex action $I$ does predict classical spacetimes (again: modulo all the difficulties adumbrated earlier!) — given that a certain technical inequality holds between the gradient of the imaginary part, $S$, of the complex action $I$, and the gradient of the real part of $I$. We note in passing that this exemplifies the idea mentioned at the end of Section 2.4, of an approximation scheme that combines in a single inequality the neglect of some quantities and a constraint on some states.

To sum up: if the relevant inequality holds (and if our other approximating and interpretative assumptions hold good!), then $S$ will be an approximate solution to the Lorentzian Hamilton-Jacobi equation for general relativity. The wave function will then be predominantly of the form $e^{i S / \hbar}$, and in that case it does define an ensemble of classical trajectories.

Of course, it must be emphasized once more that these arguments are all very heuristic since the functional integrals concerned do not exist in any proper mathematical sense. The most that can really be said is that the arguments have some validity in the context of finite-dimensional minisuperspace models.

\subsubsection{The deceptive picture: Emergence is not a process in time}

In this Subsection, we spell out the fact that the emergence of time, as treated in the Euclidean programme, and in particular by the no-boundary proposal, 
is not a process in time. Admittedly, this corollary is probably evident in the light of the discussion above. But it is worth emphasizing since a considerable proportion of the popular, and philosophical, literature about the Euclidean programme misses this point, instead taking the Euclidean manifold to be somehow 'earlier' in a temporal sense than the classical spacetime that emerges. This error results, of course, from ignoring all the interpretative difficulties, approximation assumptions etc. that we have been at pains to spell out throughout Section 5 .

In the hands of some authors, this error has misleading consequences. Namely, they argue that a treatment of the emergence of time as a process in time faces conundrums, even contradictions. (As one might guess, this is not hard to argue: for example, if it is a process there are presumably times before time emerged - surely a contradiction.) As a result, the Euclidean programme is falsely accused of facing various contradictions. Our moral will of course be that while the Euclidean programme is indeed very problematicwitness the discussion above - it does not face knockdown objections from such contradictions. ${ }^{33}$

It will be worthwhile to introduce our topic by relating the ambiguity of the word 'emergence', already noted in Section 1-process in time, versus a relation like reduction or approximation - to a distinction between two scientific disciplines: quantum gravity, and quantum cosmology. For the distinction is often ignored in the the popular, and philosophical, literature about quantum gravity.

Though closely related in various ways, these disciplines are different. In quantum gravity, one tries to reconcile general relativity and quantum theory. As we mentioned in Section 3, this reconciliation can be sought along many different avenues. But this endeavour need not necessarily involve the idea of a theory of the whole universe (let alone formulating it!). For example, some research focusses on the physics of black holes - for in the interior of a

\footnotetext{
${ }^{33}$ For these conundrums, see [28, 29] and references therein. Lack of space means that we cannot answer them seriatim here. But note that this same wrong interpretation seems to be behind Price's accusation [30] (pp.92-93) that Hawking's explanation of the arrow of time on the basis of the no-boundary proposal presupposes a temporal asymmetry; namely by applying the no-boundary proposal only at one temporal end, not both, of a temporally closed universe. Yet it is clear from Section 5.5.1 that the no-boundary proposal is in no sense 'applied at a temporal end of a universe'; indeed, nor could it be. For a philosophical review of the arrow of time in quantum cosmology, we recommend 31].
} 
black hole, the very strong gravitational field means that quantum gravity effects are expected to be important. Other programmes, such as superstring theory, concentrate on finding a role for gravity as part of a unified theory of all the fundamental forces - and theories of that type are as likely to be concerned with the interactions between particles in an accelerator as they are with the cosmos as a whole. On the other hand, in quantum cosmology one seeks a quantum-theoretical cosmology-by definition, a theory of the whole universe.

The ambiguity in 'emergence' is related to this distinction. The relation arises from the fact that in quantum cosmology, almost all work centers around theories of the very early universe: in particular, with the hope of finding a theoretical framework that avoids the mathematical singularity that is inevitably present in the account of Big Bang cosmology given by classical general relativity. And here, quantum cosmologists have made proposals that postulate a 'framework' (as we have seen it may well not deserve the name 'spacetime'!), which replaces the Big Bang singularity of classical general relativistic cosmology, and from which classical spacetime somehow 'emerges'. Probably the best-known example is the Hartle-Hawking no-boundary proposal. ${ }^{35}$

But this 'emergence' is not a process in time: Hartle and Hawking's proposed 'framework', and others such as Vilenkin's, stands in no temporal relation to classical spacetime, or any of its parts (regions or points), even very early ones.

This point is worth stressing, since three separate factors tend to obscure it. First, the word 'emergence' suggests a process in time; in which case the framework (or perhaps better: its parts) would presumably be earlier than classical spacetime (or its parts). Second, the fact that quantum cosmology focusses on the early universe makes it very tempting to think of its proposals as concerning what temporally precedes the epoch which our present theories successfully describe. Third, many popular expositions of some of the specific proposals, including the Hartle-Hawking proposal, suggest the same (wrong!)

\footnotetext{
${ }^{34}$ Here, there is indeed a link with quantum gravity: for, as in a black hole, very strong gravitational fields will make quantum gravity effects crucial in describing the Big Bang.

${ }^{35}$ But there are many other proposals: one, contemporaneous with the Hartle-Hawking proposal, and cast in the language of geometrodynamics, is Vilenkin's so-called 'tunnelling' proposal. The original paper is [32]; for a non-technical introduction see [33]; a nontechnical introduction to the competing Hartle-Hawking scheme is [34].
} 
idea.

Thus one often sees a picture in which a cone-like spacetime structure (representing a cosmological solution of classical general relativity) is attached to a spherical shape that represents a Euclidean 4-manifold. This erroneously suggests that the bottom sphere is straightforwardly earlier than the classical cosmology represented by the open cone in the top half of the figure.

But the 4-manifold is not earlier: there is no temporal relation between the two halves represented in the figure (or their parts)! Our discussion throughout Section 5 has given several reasons why not. But let us rehearse some of the more significant ones. In effect, they fall into two groups: the first group specifically concerns the no-boundary proposal; the second group returns us to the interpretative difficulties in common between the Euclidean programme and the quantum geometrodynamics of Sections 5.2-5.4-in particular, the cluster of problems called 'the problem of time'.

So first, recall that the no-boundary proposal involves a sum over different manifolds $\mathcal{M}$. So there is in any case no single bottom half-sphere, as the figure suggests - just as there is no single trajectory followed in a functionalintegral approach to elementary wave mechanics.

Second, the fact that a quantum tunnelling amplitude can be given to a good approximation by $e^{-I / \hbar}$ where $I$ is the value of the action of a solution of the classical dynamical equations with an imaginary time, does not mean that this solution has any ontological status in the quantum theory. The analogue of this point in the Hartle-Hawking proposal is, of course, the facts that the proposed 4-manifold has a Euclidean, not Lorentzian metric-and that in general relativity, a Euclidean spacetime has no more physical meaning than does an imaginary-time trajectory in normal physics. In fact, the interpretative difficulties become, if anything, even more obscure if we use (as we must) a complex metric.

To this, one might well object that one can make sense of the 4-manifold being earlier, in terms of the topological-differential, rather than metric, structure (for example, Section 3 of 28]). For example, one could easily define (regardless of metric structure) curves that start in the Lorentzian region of the manifold and enter the Euclidean region. But here we meet the second group of reasons, relating to such issues as the problem of time. However, rather than listing some of those issues again, we will venture a general statement why Hartle and Hawking's 4-manifold has no temporal relations 
to classical spacetime.

Namely, their entire proposal - the Euclidean manifold, and other postulates described above - is intended as a way of selecting a single wavefunctional $\Psi[h]$ from the set of all solutions to the Wheeler-DeWitt equation: (imagining for the sake of the argument, that this equation is well-defined).

This selection has various merits; (as well as various problems, both conceptual and mathematical). But it does not (and is not intended to) solve the problem of time; nor does it give an account of the emergence of time. That is: even if it overcame all its own problems, it would give us nothing more (nor less!) than a wave-functional of the universe obeying the Wheeler-DeWitt equation. The problem of time outlined in Section 5.2.2 here, principally the problem of finding a time with which to make sense of the Wheeler-DeWitt equation - would still have to be addressed. So would the related issues of the emergence of time: here, principally the issue, discussed in Sections 5.3-5.5.2, of how to get a classical spacetime (a temporal evolution of a single sequence of 3-geometries) from this $\Psi[h]$.

To put the point in terms of the figure of a cone emerging from a sphere: it deceptively suggests that the only conceptual obstacle to joining a classical spacetime to the Hartle-Hawking 4-manifold is the transition between the Euclidean and Lorentzian metrics. But really, there are several, subtly related obstacles: both the problem of time, and the issue of the emergence of time, lie between the two halves of the figure! ${ }^{56}$

\subsubsection{Coda: Avoiding a boundary condition?}

Finally, we turn to an aspect of the no-boundary proposal that does not fall under our theme of the emergence of time, but which is of such interest (and which has received such discussion) that we must address it, albeit briefly.

We have in mind how, if at all, the no-boundary proposal bears on the contrast between on the one hand, the laws of a physical theory given by differential equations, or equivalently their solution space; and on the other, boundary conditions, which fix one solution in the equations' solution space.円

\footnotetext{
${ }^{36}$ Though the no-boundary proposal itself does not address these obstacles, we emphasise that quantum cosmology as a discipline has of course tried to do so.

${ }^{37}$ This contrast is of course a special case of a broader contrast between the laws of a theory, which even in physics are sometimes not given by differential equations: for example, symmetry principles; and statements of particular fact, which even in physics are some-
} 
Interpretatively, one takes the former to provide the description and explanation of the possible patterns of behaviour of the system in question; the latter determine, and 'explain' the individual case.

Laws and explanation are central controversial issues in philosophy of science, which of course we cannot enter here. But we only need two uncontroversial points. First, there is a strong temptation to think of the laws (in our special case: differential equations) as being truly explanatory, while the statements of particular fact (boundary conditions) are not explanatory, because they are matters of 'mere happenstance'. Second, this thought is indeed questionable! After all, one can explain a particular fact; and one can ask for an explanation of a law. Agreed, the explanation of a particular fact will typically invoke another such fact (usually an earlier one, as in what philosophers call 'causal explanation'), and one can ask for an explanation of that fact - so that a regress beckons, and one is tempted to think that one must eventually accept a 'mere happenstance'. But similarly, when one can asks for an explanation of a law, the explanation will typically invoke another such law (relating them deductively, or by the one being a limiting case of the other), and one can ask for an explanation of that law - so again a regress beckons, and apparently one must eventually accept a 'mere happenstance' of laws also.

These two points lead directly to how the no-boundary proposal bears on the contrast between laws and boundary conditions. In essence: the noboundary proposal is advocated as promising a stop to the regress of boundary conditions, without having to settle on one as 'mere happenstance'namely, by avoiding having a boundary condition at all!

This proposal for a quantum cosmological wave-function that avoids boundary conditions (in the traditional sense) in a theoretically motivated way, is certainly very remarkable. And we ourselves find it aesthetically pleasing. But we needs must recall that it is not the only such proposal for a 'wavefunction of the universe': Vilenkin's is the best-known, and indeed contemporaneous, proposal [32]. Of course, we cannot ask the no-boundary proposal to itself rule out other such proposals, and in that very strong sense 'secure

times not given by numerical values of a function, as boundary conditions are. We should also note that a linguistic formulation of this contrast can break down. For example, in the familiar case of fixing the solution of a first-order ordinary differential equation by specifying an 'initial' boundary condition, the differential equation plus boundary condition can be combined in a single integral equation. 
uniqueness'. But we should note that at present, both these proposals and indeed others are not ruled out; and in that sense, the science of quantum cosmology as a whole has not avoided the 'regress of happenstance'. To do so would of course be a very tall order: we do not mean this point as a criticism. Indeed, in our opinion what matters is not the prospects for eventually doing so, but rather the role of the no-boundary proposal, and similar proposals, in understanding that alluring idea - the nature, and emergence, of time.

\section{Conclusion}

To sum up our discussion: we have discussed two programmes within quantum gravity, quantum geometrodynamics and the Euclidean programme. We have focussed on the merits and difficulties of their treatments of the emergence of time (more precisely: the spacetime of general relativity).

Broadly speaking, our conclusion is twofold. First, we concede that there are large conceptual difficulties in both programmes' attempts to have classical spacetime 'emerge' (as well as ferocious technical difficulties). But second, more positively, we see no knock-down conceptual errors, or philosophical howlers, in these attempts, as they have been developed so far. In that sense, there are good prospects for work for the future.

Acknowledgements - Chris Isham thanks the Mrs L.D. Third Charitable Settlement for financial assistance during during part of the course of this work. One of us (JB) is very grateful to Katinka Ridderbos for discussions and comments.

\section{References}

[1] G. Belot and J. Earman. Pre-socratic quantum gravity. In C. Callender and N. Huggett, editors, Physics Meets Philosophy at the Planck Scale. Cambridge University Press, Cambridge, 1999.

[2] J.B. Hartle and S.W. Hawking. Wave function of the universe. Phys. Rev., D28:2960-2975, 1983.

[3] E. Nagel. The Structure of Science. Routledge and Kegan Paul, London, 1961. 
[4] M. Spector. Concepts of Reduction in Physical Science. Temple University Press, Philadelphia, 1978.

[5] A Khinchin. Mathematical Foundations of Statistical Mechanics. Dover, New York, 1949.

[6] P. Smith. Modest reductions and the unity of science. In Reduction, Explanation and Realism, pages 19-43. Oxford University Press, Oxford, 1992.

[7] P. Feyerabend. Explanation, reduction and empiricism. In H. Feigl and G. Maxwell, editors, Minnesota Studies in Philosophy of Science vol. 3. University of Minnesota Press, Minneapolis, 1962. Reprinted in his (1981) Realism, Rationalism and Scientific Method, (Cambridge University Press, Cambridge).

[8] G. Hellman and F. Thompson. Physicalism: Ontology, determination and reduction. Journal of Philosophy, 72:551-564, 1975.

[9] M. Wilson. What is this thing called pain? - the philosophy of science behind the contemporary debate. Pacific Philosophical Quarterly, 66:227-267, 1985 .

[10] A. Compagner. Thermodynamics as the continuum limit of statistical mechanics. American Journal of Physics, 57:106-117, 1989.

[11] J. Butterfield and C.J. Isham. The Philosophical Challenge of Quantum Gravity. 1999.

[12] C.J. Isham. Structural issues in quantum gravity. In General Relativity and Gravitation: GR14, pages 167-209. World Scientific, Singapore, 1997.

[13] W. Clifford. On the hypotheses which lie at the bases of geometry. $\mathrm{Na}$ ture, 8:14-17, 36-37, 1873. Translation of Riemann's Habilitationschrift (1854).

[14] C.J. Isham. Quantum topology and quantization on the lattice of topologies. Class. Quan. Grav., 6:1509-1534, 1989. 
[15] R.D. Sorkin. Posets as lattice topologies. In B. Bertotti, F. de Felice, and A. Pascolini, editors, General Relativity and Gravitation: Proceedings of the GR10 Conference. Volume I, pages 635-637. Consiglio Nazionale Delle Ricerche, Rome, 1983.

[16] R.D. Sorkin. Spacetime and causal sets. In J.C. D'Olivo, E. NahmadAchar, M. Rosenbaum, M.P. Ryan, L.F. Urrutia, and F. Zertuche, editors, Relativity and Gravitation: Classical and Quantum, pages 150-173. World Scientific, Singapore, 1991.

[17] A. Maidens. Symmetry groups, absolute objects and action principles in general relativity. Studies in History and Philosophy of Modern Physics, 29B:245-272, 1998.

[18] D. Giulini, E. Joos, C. Kiefer, J. Kupsch, I.-O. Stamatescu, and H.D. Zeh. Decoherence and the Appearance of a Classical World in Quantum Theory. Springer, Berlin, 1996.

[19] A. Kent. Against many world interpretations. International Journal of Modern Physics, A5:1745-1762, 1990.

[20] J. Butterfield. Worlds, minds and quanta. Aristotelian Society Supplementary Volume, 69:113-158, 1995.

[21] J. Butterfield. Whither the minds? British Journal for the Philosophy of Science, 47:200-221, 1996.

[22] K. Kuchař. Time and interpretations of quantum gravity. In Proceedings of the 4th Canadian Conference on General Relativity and Relativistic Astrophysics, pages 211-314. World Scientific, Singapore, 1992.

[23] C.J. Isham. Canonical quantum gravity and the problem of time. In Integrable Systems, Quantum Groups, and Quantum Field Theories, pages 157-288. Kluwer Academic Publishers, London, 1993.

[24] W. Unruh and R.M. Wald. Time and the interpretation of quantum gravity. Phys. Rev., D40:2598-2614, 1989.

[25] D. Lewis. A subjectivist's guide to objective chance. In D. Lewis, editor, Philosophical Papers vol. II. Oxord University Press, Oxford, 1986. 
[26] K. Kuchař. The problem of time in canonical quantization. In A. Ashtekar and J. Stachel, editors, Conceptual Problems of Quantum Gravity, pages 141-171. Birkhäuser, Boston, 1991.

[27] S.W. Hawking. Particle creation by black holes. Comm. Math. Phys., 43:199-220, 1975.

[28] Q. Smith. The ontological interpretation of the wave function of the universe. The Monist, 80:160-185, 1997.

[29] R. Deltete and R. Guy. Emerging from imaginary time. Synthese, 108:185-203, 1996.

[30] H. Price. Time's Arrow and Archimedes' Point. Oxford University Press, Oxford, 1996.

[31] K. Ridderbos. The arrow of time in quantum cosmology. 1999. In preparation.

[32] A. Vilenkin. Quantum cosmology and the initial state of the universe. Phys. Rev., D39:888-897, 1988.

[33] C.J. Isham. Quantum theories of the creation of the universe. In R. J. Russell, N. Murphy, and C.J. Isham, editors, Quantum Cosmology and the Laws of Nature, pages 49-90. University of Notre Dame Press, Notre Dame, 1993.

[34] C.J. Isham. Creation of the universe as a quantum tunnelling process. In R. J. Russell, W. Stoeger, and G.V. Coyne, editors, Our Knowledge of God and Nature: Physics, Philosophy and Theology, pages 374-408. University of Notre Dame Press, Notre Dame, 1988. 